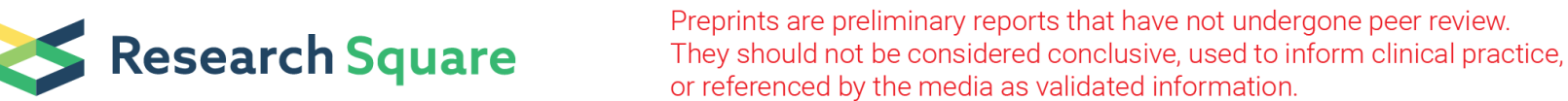

\section{Integrated Transcriptome and Proteome Analysis Reveals Complex Regulatory Mechanism of Cotton in Response to Salt Stress}

\section{Lin Chen}

Huazhong Agriculture University

Heng Sun

Huazhong Agriculture University

Jie Kong

Xinjiang Academy of Agricultural Sciences

Haijiang Xu

Xinjiang Academy of Agricultural Sciences

Xiyan Yang ( $\nabla$ yxy@mail.hzau.edu.cn )

Huazhong Agricultural University: Huazhong Agriculture University

\section{Research}

Keywords: Association analysis, Cotton, Proteome, Salt stress, Transcriptome

Posted Date: November 19th, 2020

DOI: https://doi.org/10.21203/rs.3.rs-107670/v1

License: (c) (1) This work is licensed under a Creative Commons Attribution 4.0 International License. Read Full License

Version of Record: A version of this preprint was published at Journal of Cotton Research on April 25th, 2021. See the published version at https://doi.org/10.1186/s42397-021-00085-5. 


\section{Abstract}

\section{Background}

Soil salt stress seriously restricts the yield and quality of cotton worldwide. To investigate the molecular mechanism of cotton response to salt stress, a main cultivated variety Gossypium hirsutum L. acc. Xinluzhong 54 was used to perform transcriptome and proteome integrated analysis.

Results

Through transcriptome analysis of cotton treated with salt stress for $0 \mathrm{~h}$ (T0), $3 \mathrm{~h}$ (T3) and $12 \mathrm{~h}$ (T12), we identified 8,436, 11,628 and 6,311 differentially expressed genes (DEGs) inT3 / T0, T12 / T0 and T12 / T3, respectively. A total of 459 differentially expressed proteins (DEPs) were identified by proteomic analysis, of which 273, 99 and 260 DEPs were identified in T3 / T0, T12 / T0 and T12 / T3, respectively. Metabolic pathways, biosynthesis of secondary metabolites, photosynthesis and plant hormone signal transduction were the main enrichment pathways by annotation of DEGs or DEPs. Detail analysis of the DEGs or DEPs revealed that complex signal pathways, such as ABA and JA signal, calcium signal, MAPK signal cascade, transcription factors, followed by activation of antioxidant and ion transporters, were identified to participate in regulating salt response in cotton.

Conclusions

Our results not only contribute to understand the mechanism of cotton response to salt stress, but also provide nine candidate genes, which might be used for molecular breeding to improve salt-tolerance in cotton.

\section{Background}

Soil salinity caused by high concentration of salt ions in soil, which seriously restricts the production of agriculture. More than 800 million hectares of land and $20 \%$ of the arable land throughout the world are affected by salt stress, which will continue to deteriorate (Munns and Tester 2008; Al Murad et al., 2020). It was showed that the problem of soil salinity become more and more serious in China. For that the area of saline-alkali land in China is about 100 million hectares. Moreover, the increased area of secondary salinization in the western region and Yellow River Delta are 1.5-2 and 1 hundred thousand mu every year, respectively. The high concentration of salt ions can change soil structure, reduce soil permeability and water conductivity, trigger different level of effects on plants, such as osmotic stress, ion cytotoxicity, nutritional deficiency, and oxidative stress (Mahajan and Tuteja 2005; Muchate et al., 2016; Zelm et al., 2020). Osmotic stress reduces water uptake and leads to water deficit in plants, which occurs in the early stage of salt stress. Ion cytotoxicity is caused by the high concentration of salt ions $\left(\mathrm{Na}^{+}\right)$in the cytoplasm, and it disrupts the uptake of other ions into plant cells and obstructs some metabolic pathways, such as photosynthesis (Zhang et al., 2019; Wang et al., 2020). Osmotic stress and ionic stress 
can cause secondary stress in plants, for example, the high concentration of reactive oxygen species will cause damage to some cell structures and biological macromolecules (Yang and Guo 2018).

The salt tolerance of plants depends on their ability to extract water and nutrients from saline soils and to avoid excessive accumulation of salt ions in plant tissues (Zorb et al., 2019). Most of crop plants are saltsensitive, and these crops planted in moderate salt stress conditions will reduce yields severely (Zorb et al., 2019; Park et al., 2016). Therefore, analyzing the mechanism of plant response to salt stress and cultivating new salt-tolerant crop varieties are very important to ensure the sustainability of agricultural production and food security.

Cotton (Gossypium) is the most important fiber cash crop, and China is the largest producer and consumer of cotton in the world. Xinjiang is the main cotton producing area in China, and more than $32.6 \%$ cultivated land in Xinjiang is affected by different degrees of soil salinization stress, which seriously threatens the safety of cotton production. It is very important to improve salt tolerance on the basis of ensuring high yield and quality in cotton. Transcriptome and proteome have made some progress in revealing the mechanism of salt tolerance and identification of candidate genes in cotton (Peng et al., 2014; Guo et al., 2015; Gong et al., 2017; Li et al., 2015). However, as a multi-functional complex system, there are multi-level regulatory relationships between gene transcription and translation. It is necessary to monitor the gene expression of RNA and protein simultaneously. Fortunately, the development of integrated transcriptome and proteome makes this research strategy possible (Trevisan et al., 2015; Chen et al., 2015; Wang et al., 2014; Peng et al., 2018).

In the present study, an integrated transcriptome and proteome analysis were performed to deeply investigate the molecular mechanism of cotton in response to salt stress. There were 15,822 DEGs and 459 DEPs were identified in mRNA transcription and protein expression level, respectively, and, a total of 164 associated DEGs and DEPs were identified. And some important biological pathways were elucidated. Furthermore, 9 candidate genes were identified by integrating proteomic and transcriptomic profiles. Our results will further enrich the molecular mechanism of cotton in response to salt stress.

\section{Materials And Methods}

\section{Plant materials and treatments}

The widely planted upland cotton variety Xinluzhong 54 (Gossypium hirsutum L. acc.) was used in the research, and it was provided by Xinjiang Academy of Agricultural Sciences (Additional file 1: Fig. S1). Cotton seedlings were cultured in Hoagland solution under $16 \mathrm{~h}$ light/8 h dark conditions. For salt stress treatment, $250 \mathrm{mM} \mathrm{NaCl}$ was added to the solution at two-leaf stage seedlings to simulate salt stress. The leaves were collected at $0 \mathrm{~h}$ (T0), $3 \mathrm{~h}$ (T3) and $12 \mathrm{~h}$ (T12) for subsequent RNA and protein isolations.

\section{RNA-seq and ITRAQ}


Total RNAs were extracted using the RNAprep Pure Plant Kit (Cat. \#DP441, TIANGEN). RNA-Seq was conducted using Illumina Hiseq platform (BGI Tech, Shenzhen, China). After remove the low-quality, adaptor-polluted and high content of unknown base (N) reads, Gossypium hirsutum (Var. TM-1) genome sequences were used as references for read mapping and gene annotation (Zhang et al., 2015). The gene expression levels for each sample were calculated with RSEM, the differentially expressed genes (DEGs) were detected with DEseq2 (Fold change $\geq 2$, adjusted $P$ value $\leq 0.05$ ).

For iTRAQ analysis, leaf samples were sent to BGI company (BGI Tech, Shenzhen, China) for quantitative analysis, the main processes include protein extraction, protein digestion, peptide labeling, peptide fractionation and LC-MS / MS. Quantitative analysis was performed by IQuant software (Wen et al., 2014). T3 / T0, T12 / T0 and T12 / T3 were set as comparison groups, the differentially expressed proteins (DEPs) were identified according to fold change $>1.2$ (or $<0.83$ ) and Q-value $<0.05$. Both RNASeq and ITRAQ experiments were performed with three biological replicates.

\section{Functional enrichment analysis}

KEGG (Kyoto Encyclopedia of Genes and Genomes) and GO (Gene Ontology) functional enrichment analysis of DEGs and DEPs were performed using "Gene-list Enrichment" program in KOBAS3.0 database (http://kobas.cbi.pku.edu.cn/anno_iden.php) (Xie et al., 2011). Transcription factors were identified by PlantTFDB 4.0 (http://planttfdb.cbi.pku.edu.cn/). The protein interaction networks were predicted by STRING (version 11.0), and were visualized by Cytoscape software (3.4.0). The gene/protein expression patterns were showed by heatmap with the expression values normalized by Genesis software.

\section{qRT-PCR analysis}

High-quality cDNA was obtained by reverse transcription using SuperScript III Reverse Transcriptase in accordance with the manufacturer's instructions (Cat. No.18080-093, Invitrogen). Gene expression was detected using an ABI Prism 7500 system (Applied Biosystems). GhUBQ7 (GenBank accession No. DQ116441) was used as the internal control. Gene-specific primers for qRT-PCR were designed according to the cDNA sequences using Primer Premier 5.0 software and synthesized commercially (Genscript Bioscience) (Additional file 1: Table S1).

\section{Results}

\section{Identification of DEGs and DEPs during cotton response to salt stress}

The seedlings of Xinluzhong 54 (Gossypium hirsutum L. acc.) were cultured in Hoagland solution and $250 \mathrm{mM} \mathrm{NaCl}$ was added to simulate salt stress. Leaves at two-leaf stage were used for transcriptome and proteome analysis, the workflow of experimental design and analysis was shown in Fig. 1a. Three samples each with three biological replicates were identified using Illumina Hiseq platform and iTRAQ system. 
The average output data of each sample from RNA-Seq was $6.64 \mathrm{~Gb}$. The average Q20 and Q30 of each sample were $97.77 \%$ and $93.65 \%$, respectively. The clean reads were mapped to the reference genome of Gossypium hirsutum (TM-1), and the average alignment rate of each sample is $82.83 \%$ (Additional file 1: Table S2). DEseq2 algorithm were used to detect the DEGs with the standard of fold change $\geq 2$ and $P$ value $\leq 0.05$. There were 8,436 (4,123 up-regulated, 4,313 down-regulated), 11,628 (4,649 up-regulated, 6,979 down-regulated) and 6,311 (2,169 up-regulated, 4,142 down-regulated) DEGs were identified in T3 / T0, T12 / T0 and T12 / T3, respectively (Fig. 1b). DEGs distributed more in D sub-genome (53\%) than A sub-genome. And more DEGs distributed on chromosomes A05 and D05, while less distributed on A04 and D03 (Additional file 1: Fig. S2a). Twenty-five genes were randomly selected to evaluate the RNA-Seq results by qRT-PCR, there was a significant correlation between these two groups data $\left(R^{2}=0.874\right)$ (Additional file 1: Fig. S2b). It indicated that the sequencing data of the RNA-Seq had high reliability, and could be used for subsequent analysis. Venn diagram showed that 5,402, 3,945 and 2,342 common DEGs were detected in T3 / T0 and T12 / T0, T12 / T0 and T12 / T3, T3 / T0 and T12 / T3, respectively, with 1,136 common DEGs shared by the three comparisons (Fig. 1c). The 1,136 common DEGs showed upregulated and down-regulated expression patterns by calculate hierarchical clustering analysis, with upregulated genes enriching in galactose metabolism, biosynthesis of secondary metabolites, metabolic pathways, valine, leucine and isoleucine degradation, glucosinolate biosynthesis, and down-regulated genes in metabolic pathways, carbon fixation in photosynthetic organisms, biosynthesis of secondary metabolites, carbon metabolism and flavonoid biosynthesis (Additional file 1: Fig. S3a).

A total of 459 DEPs were identified by iTRAQ, and 273 (185up-regulated, 88 down-regulated), 99 (38 upregulated, 61 down-regulated) and 260 (106 up-regulated, 154 down-regulated) DEPs were identified in T3 / T0, T12 / T0 and T12 / T3, respectively (Fig. 1d). All DEPs encoding genes equally distributed on A subgenome and D sub-genome, and more DEPs distributed on chromosomes A05 and D05. Venn diagram showed that 18, 127 and 35 common DEPs in T3 / T0 and T12 / T0, T3 / T0 and T12 / T3, T12 / T0 and T12 / T3, respectively (Fig. 1e). There were seven common DEPs in three groups, three proteins (Gh_A02G0551, Gh_A07G0867, Gh_D08G1902) were up-regulated at 3 h, two proteins (Gh_D02G2126, Gh_A03G1705) were down-regulated at $3 \mathrm{~h}$ and up-regulated at $12 \mathrm{~h}$, and one protein (Gh_Sca010764G01) was up-regulated at $3 \mathrm{~h}$ and $12 \mathrm{~h}$, one protein (Gh_D11G1672) was down-regulated at $3 \mathrm{~h}$ and $12 \mathrm{~h}$.

\section{Integration of proteomic and transcriptomic profiles}

To investigate the multi-level regulation of gene expression under salt stress, an integrative analysis of the transcriptome and proteome was performed in this study. Results showed that 77,70 and 47 associated DEGs and DEPs were identified in T3 / T0, T12 / T0 and T12 / T3, respectively (Fig. 2a, Additional file 1: Table S3). In T3 / T0, 31 and 46 genes showed the same and opposite expression patterns at transcriptional level and protein level, respectively. In T12 / T0, 62 and 8 genes showed the same and opposite expression patterns at these two levels, respectively. In T12 / T3, 31 and 16 genes showed the same and opposite expression patterns at these two levels, respectively (Fig. 2a). These 
results suggested that genes in response to salt stress may undergo complex regulation at the transcriptional and protein levels.

Venn diagram showed that nine common associated genes (Gh_D08G1902\Gh_A07G0867

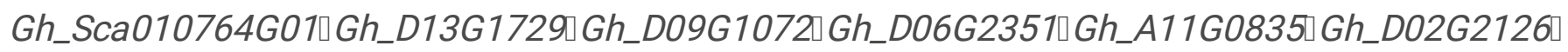
Gh_A03G1705) were found in theT3 / T0 and T12 / T0, 11 associated genes (Gh_D11G1672

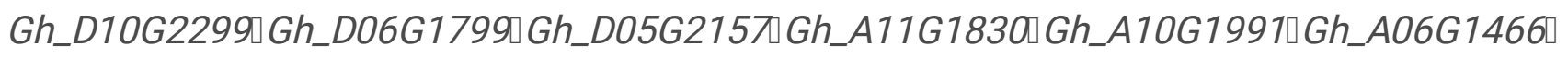
Gh_A05G1452 Gh_A01G1839]Gh_D02G2126ロGh_A03G1705) were identified in the T12 / T0 and T12 /

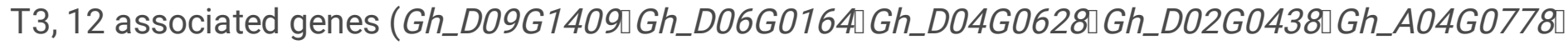

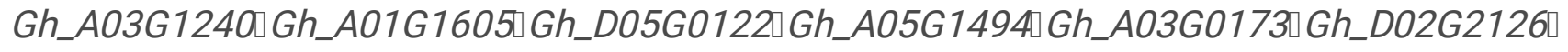
Gh_A03G1705) were identified in the T3 / T0 and T12 / T3 (Fig. 2b). All associated genes were listed in Additional file 1: Table S4. Spearman correlation analysis showed that a poor correlation between the expression changes of these associated DEGs and DEPs was observed ( $R=-0.37-0.71)$ (Fig. 2c).

\section{Functional enrichment analysis of DEGs and DEPs}

KEGG analysis was performed to study the functional enrichment of up-regulated and down-regulated DEGs in each comparison group. For up-regulated DEGs, there were 21, 30 and 4 pathways were enriched in T3 / T0, T12 / T0 and T12 / T3, respectively (Additional file 1: Fig. S3b). Plant hormone signal transduction, biosynthesis of secondary metabolites and metabolic pathways occurred simultaneously in these three groups. For down-regulated DEGs, there were 17, 22 and 25 pathways were enriched in T3 / T0, T12 / T0 and T12 / T3, respectively (Additional file 1: Fig. S3c). Ribosome was the most significant enrichment pathway in T3 / T0, and metabolic pathways was significantly enriched in T12 / T0 and T12 / T3.

For up-regulated DEPs, there were 8, 2 and 14 pathways were enriched in T3 / T0, T12 / T0 and T12 / T3, respectively (Corrected $P \leq 0.01$ ) (Additional file 1: Fig. S4a). Photosynthesis and pyruvate metabolism were the common pathways in these three groups. Glyoxylate and dicarboxylate metabolism, ribosome, carbon fixation in photosynthetic organisms and citrate cycle (TCA cycle) were just significantly enriched in T3 / T0. In addition, ten pathways were just enriched in T12 / T3, such as endocytosis, alpha-Linolenic acid metabolism and glycerophospholipid metabolism. For down-regulated DEPs, there were 11, 13 and 9 pathways were enriched in T3 / T0, T12 / T0 and T12 / T3, respectively (Additional file 1: Fig. S4b). Metabolic pathways and biosynthesis of secondary metabolites were the common pathways in these three groups. In addition, selenocompound metabolism, biosynthesis of amino acids and porphyrin and chlorophyll metabolism were commonly enriched in T3 / T0 and T12 / T0, photosynthesis and ribosome were commonly enriched in T3 / T0 and T12 / T3, flavonoid biosynthesis and carbon fixation in photosynthetic organisms were commonly enriched in T12 / T0 and T12 / T3.

KEGG analysis showed that these associated genes/proteins enriched in multiple pathways (Corrected $P$ value $\leq 0.05$ ) (Fig. 2d). We found some DEGs showed the same change patterns at transcriptional and 
protein levels and were enriched in some pathways, such as flavonoid biosynthesis (Gh_D08G1902, Gh_A10G1079, Gh_A05G1424, Gh_A12G0549), porphyrin and chlorophyll metabolism (Gh_A09G1143, Gh_A05G3766, Gh_D01G1828, Gh_A10G0282, Gh_A10G2274), thiamine metabolism (Gh_A06G1269, Gh_A05G1432, Gh_D05G1605), biosynthesis of amino acid (Gh_D08G2317, Gh_A13G1464, Gh_D13G1473, Gh_D06G1578, Gh_D01G1099, Gh_D05G2157, Gh_D09G0576).

\section{Disturbance of energy metabolism caused by salt stress}

The carbohydrate metabolism pathways and photosynthesis were very important for the storage and utilization of energy in plants. Here, the changes of DEGs and DEPs which involved in these two biological processes were analyzed. Treated with salt stress, cotton genes exhibited strong expressing involved in the glycolysis/ gluconeogenesis pathways. The expression of GhPFK3 (Gh_A05G0198), GhPKP2 (Gh_A08G0871) and GhPKP1 (Gh_A10G1036) were up-regulated by salt stress, and the gene GhHXK3 (Gh_D06G0003) encoding hexokinase (HK) was down-regulated by salt stress, the transcriptional level of was down-regulated by 4.52 times and 10.37 times at $3 \mathrm{~h}$ and $12 \mathrm{~h}$, respectively (Fig. 3a). The protein abundance of enolase PGH1 (Gh_Sca286293G01), fructose-bisphosphate aldolase FBA5 (Gh_D13G1361), pyruvate dehydrogenase subunit (Gh_A12G1299) and triosephosphate isomerase (Gh_D01G1600) showed significantly increased at $3 \mathrm{~h}$ after salt stress (Additional file 1: Fig. S5a). The acetaldehyde dehydrogenase protein (Gh_D06G1578) increased significantly at $12 \mathrm{~h}$, hexokinase HK (Gh_A13G1742) and ethanol dehydrogenase (Gh_A01G1605) protein decreased at $3 \mathrm{~h}$.

The expression down-regulation was observed in DEGs encoding isocitrate dehydrogenase (IDH), GhCICDH (Gh_A11G1562), pyruvate dehydrogenase E1 subunit, GhMAB1 (Gh_A12G1299) and malate dehydrogenase, GhMMDH2 (Gh_A01G04040), GhMMDH1 (Gh_A04G0320) involved in tricarboxylic acid (TCA) cycle (Fig. 3b). And the protein abundance of malate dehydrogenase (Gh_D02G0438, Gh_A02G0386, Gh_D05G3328) and pyruvate dehydrogenase E1 subunit (Gh_A12G1299) increased at $3 \mathrm{~h}$ after salt stress (Additional file 1: Fig. S5b). The protein abundance of succinate dehydrogenase subunit (Gh_A10G1110) and 2-oxoglutarate dehydrogenase (Gh_A05G3057) decreased at $3 \mathrm{~h}$ (Additional file 1: Fig. S5b).

Similarly, the expression of genes modulating photosynthesis were significant down regulated in cotton. such as photosystem I subunit PSAO related gene, GhPSAO (Gh_D07G1090) and light harvesting chlorophyll protein complex LHC related genes, GhLHCB1 (Gh_D06G2351), GhLHCB2 (Gh_A07G1725) and GhLHCA4 (Gh_A12G1617) (Fig. 3c). The protein abundances of most DEPs involved in photosynthesis were increased under salt stress (Additional file 1: Fig. S5c). For example, photosystem II subunit PSBO (Gh_D11G1897), PSBP (Gh_A05G3293, Gh_D04G0311) and PSBQ2 (Gh_A10G2195) proteins increased at $3 \mathrm{~h}$ under salt stress. The abundance of photosystem I related proteins psaA (Gh_A01G1364, Gh_A09G0964) and psaB (Gh_A12G1136, Gh_Sca005646G01) decreased after $3 \mathrm{~h}$ of salt stress, but increased at $12 \mathrm{~h}$. Taken together, these results suggested that salt stress can cause the 
changes in expression of cotton genes involved in arbohydrate metabolism pathways and photosynthesis pathways, to facilitate the tolerance and sustain cotton survive in salt stress.

\section{Signal transduction in response to salt stress in cotton Plant hormone signal transduction}

Plant hormones play an important role in the regulation of plant salt stress resistance. Here, the expression changes of key node genes in different hormone signal pathways were analyzed (Fig. S6). $A B A$ and JA hormone signals are important for plant to resistant salt stress. Most of the genes involved in ABA signal pathway, such as PP2C, SnRK2 and ABF, were up-regulated by salt stress (Fig. 4a). We speculated that the continuous activation of $A B A$ signal may play an important role in cotton response to salt stress. $A B F$ transcription factors play an important role in regulating $A B A$ signal transduction. There were 14 genes encoding $A B F$ transcription factors were identified, both of them were up-regulated by salt stress (Fig. 4a). Nine ABF genes (Gh_D05G2495, Gh_A05G2234, Gh_D12G0214, Gh_A05G1751, Gh_D05G1946, Gh_D02G0909, Gh_A03G1664,Gh_A03G2095, Gh_D02G2079) were continuously induced by salt stress during 0-12 h, and the maximum expression of four genes (Gh_A12G0212, Gh_D13G0400, Gh_A13G0355, Gh_A13G2006) were detected at $3 \mathrm{~h}$ (Fig. 4a).

The JAR gene of JA pathway encodes a jasmonic acid-amino synthase, which could catalyze the formation of bioactive jasmonic acid-isoleucine (JA-lle). The JAR1 coding gene Gh_A08G1120 and Gh_D08G1403 were significantly up-regulated in T3 / T0, we speculated that salt stress might induce JAlle synthesis and activate JA signal in early stage (Fig. 4b). MYC2 is an important transcription factor in JA signal pathway. We identified two genes encoding MYC2 transcription factors, Gh_D08G1707 was significantly induced by salt stress and showed continuous expression patterns, however Gh_D09G1895 was down-regulated by salt stress. Furthermore, there were 21 differentially expressed JAZ genes which were identified, among of them, 18 genes were up-regulated (Fig. 4b).

\section{Transcription factor}

Transcription factor mediated transcriptional regulation plays an important role in plant response to abiotic stress. There were 696 (429 up-regulated, 267 down-regulated), 926 (492 up-regulated, 434 downregulated) and 532 (232 up-regulated, 300 down-regulated) transcription factor were identified in T3 / T0, T12 / T0 and T12 / T3, respectively (Fig. S7a and b). Among these transcription factors, NAC, ERF, MYB, bZIP and WRKY family genes accounted for a large proportion. Some transcription factors with the spatio-temporal expression patterns, about $32 \%$ and $49 \%$ transcription factors were only differentially expressed at $3 \mathrm{~h}$ and $12 \mathrm{~h}$ after salt stress, respectively (Additional file 1: Fig. S7a and b). Some transcription factors that response to salt stress revealed by previously studies were also detected in this study (Additional file 1: Fig. S7c).

\section{CBL-CIPK signal cascade}


CBL-CIPK signal system can participate in the process of plant stress response by sensing calcium ion to regulate intracellular signal transduction. Two genes encoded CBL were identified, GhCBL2 (Gh_A03G0846) and GhCBL3 (Gh_A13G1099), these two genes were significantly up-regulated by salt stress. There were $43 \mathrm{CIPK}$ genes were identified, and most of genes were up-regulated by salt stress (Fig. 4C). We predicted the potential protein interaction models between these CBL and CIPK proteins through the STRING database. Results showed that these two CBL proteins could interact with multiple CIPK proteins, respectively (Additional file 1: Fig. S8a). Furthermore, some CBL and CIPK genes with similar expression patterns under salt stress, such as CBL2 and CIPK8 (Gh_A05G3756, Gh_A06G1872, Gh_D05G2084), both of them were continuously induced by salt stress.

\section{Mitogen-activated protein kinase signal cascade}

Mitogen-activated protein kinase (MAPK) cascade pathway is composed of three kinds of serine / threonine protein kinases, MAP3K, MKK and MPK. There were 18, 4 and 17 genes encoded MAP3K, MKK, MPK were identified, respectively (Fig. 4d). Most of the MAPK pathway genes were up-regulated by salt stress at $3 \mathrm{~h}$. The potential protein interaction models were also predicted through the STRING database. Two up-regulated genes Gh_A07G0124 and Gh_D07G2384 which encoding GhMKK2, showed widely predicted interaction models with up-stream MAP3K proteins and down-stream MPK proteins. We speculated that GhMKK2 might play an important role in regulating cotton response to salt stress by MAPK cascade pathway (Additional file 1: Fig. S8b).

\section{Antioxidant and ion transport systems}

Salt stress cause the imbalance of reactive oxygen species (ROS) homeostasis and activate the ROS scavenging mechanism. NADPH oxidase is involved in the production of ROS in plants under stress. Four genes (GhRBOHA; Gh_A02G1791, Gh_A12G2669, Gh_D03G0688 and Gh_D12G2750) encoding NADPH oxidase respiratory burst oxidase homolog $A$ (RBOHA) were up-regulated by salt stress (Fig. 5a). Consistently, dramatic expression up-regulation was detected in genes encoding catalase (GhCAT2), superoxide dismutase (GhFSD2) or glutathione S-transferase (GhGSTT1, GhGSTZ1) (Fig. 5b). Our results indicated that the enzyme pathways involved by CAT, SOD and GST genes might play an important role in protecting cotton from oxidative damage caused by salt stress.

The regulation of intracellular ion homeostasis is very important for plants response to salt stress. Ion channel protein $\left(\mathrm{Na}^{+} / \mathrm{H}^{+} / \mathrm{K}^{+}\right.$transporter) are tightly linked to plant response to salt stress. Thus, we analyzed the expression of genes that encoding ion transporters. GhNHX2 (Gh_A11G2132) and GhKUP3 (Gh_D04G0700), GhKUP11 (Gh_A12G0438) encoding Na ${ }^{+} / \mathrm{H}^{+}$transporters and $\mathrm{K}^{+}$transporters respectively, were significantly up-regulated by salt stress (Fig. 5c). GhHKT1 (Gh_A08G2473) encoded $\mathrm{Na}^{+}$transporters were down-regulated under salt stress at $12 \mathrm{~h}$. Potassium channels are involved in the regulation of ion absorption and transport. And a significant up-regulated at $12 \mathrm{~h}$ by salt stress were observed in genes that encoding inward rectifying potassium channel (GhAKT1, Gh_D13G2111) and 
outward rectifying K ${ }^{+}$channel (GhTPK1, Gh_D01G0726; GhKCO5, Gh_A11G0947; GhSKOR, Gh_D05G1041) (Fig. 5d).

\section{The identification of candidate genes by integrating proteomic and transcriptomic profiles}

Based on the gene expression and functional annotation, we identified 9 candidate genes might play an important role in cotton response to salt stress, GhLEA14 (Gh_A11G0835), GhKCS6 (Gh_A03G1286), GhPRXR1 (Gh_A05G1452), GhPOD(Gh_A08G0714), GhFIB (Gh_A05G1494), GhALDH7B4 (Gh_D06G1578), GhUSP (Gh_A03G0386) and GhAVP1 (Gh_D05G0122), GhADH1 (Gh_A01G1605) (Table 1). GhLEA14 encodes late embriogenesis abundant protein and its expressive abundance was upregulated at both transcriptional and protein levels under salt stress, it might be involved in the regulation of osmotic stress and the prevention of oxidant stress in cotton response to salt stress. The peroxidase superfamily protein GhPRXR1 and GhPOD were up-regulated at transcription and protein levels by salt stress, these two genes might be involved in the hydrogen peroxide catabolic process. GhALDH7B4 encoding ethanol dehydrogenase and acetaldehyde dehydrogenase, respectively. These two genes were up-regulated at the transcriptional and protein levels under salt stress, we speculated that these two genes may be involved in the detoxification of cotton cells under salt stress. 
Table 1

Candidate genes involved in salt stress response in cotton

\begin{tabular}{|c|c|c|c|c|c|}
\hline Name & Gene_ID & Stage & $\begin{array}{l}\text { DEG } \\
\text { log2FoldChange }\end{array}$ & $\begin{array}{l}\text { DEP } \\
\text { log2FoldChange }\end{array}$ & Description \\
\hline \multirow[t]{2}{*}{ GhLEA14 } & \multirow[t]{2}{*}{ Gh_A11G0835 } & $\begin{array}{l}\text { T3 / } \\
\text { T0 }\end{array}$ & 4.53 & 0.55 & \multirow{2}{*}{$\begin{array}{l}\text { Late } \\
\text { embryogenesis } \\
\text { abundant protein }\end{array}$} \\
\hline & & $\begin{array}{l}\text { T12 / } \\
\text { T0 }\end{array}$ & 2.84 & 0.85 & \\
\hline GhKCS6 & Gh_A03G1286 & $\begin{array}{l}\text { T12 / } \\
\text { T0 }\end{array}$ & 3.57 & 1.43 & $\begin{array}{l}\text { 3-ketoacyl-CoA } \\
\text { synthase } 6\end{array}$ \\
\hline \multirow[t]{2}{*}{ GhPRXR1 } & \multirow[t]{2}{*}{ Gh_A05G1452 } & $\begin{array}{l}\text { T12 / } \\
\text { T3 }\end{array}$ & 1.72 & 0.82 & \multirow{2}{*}{$\begin{array}{l}\text { Peroxidase } \\
\text { superfamily } \\
\text { protein }\end{array}$} \\
\hline & & $\begin{array}{l}\text { T12 / } \\
\text { T0 }\end{array}$ & 1.68 & 0.77 & \\
\hline GhPOD & Gh_A08G0714 & $\begin{array}{l}\text { T3 / } \\
\text { T0 }\end{array}$ & 1.58 & 0.41 & $\begin{array}{l}\text { Peroxidase } \\
\text { superfamily } \\
\text { protein }\end{array}$ \\
\hline \multirow[t]{2}{*}{ GhFIB } & \multirow[t]{2}{*}{ Gh_A05G1494 } & $\begin{array}{l}\text { T3 / } \\
\text { T0 }\end{array}$ & 1.28 & 0.67 & \multirow[t]{2}{*}{$\begin{array}{l}\text { Fibrillin precursor } \\
\text { protein }\end{array}$} \\
\hline & & $\begin{array}{l}\text { T12 / } \\
\text { T3 }\end{array}$ & -2.38 & -0.43 & \\
\hline GhALDH7B4 & Gh_D06G1578 & $\begin{array}{l}\text { T12 / } \\
\text { T0 }\end{array}$ & 2.48 & 0.31 & $\begin{array}{l}\text { Aldehyde } \\
\text { dehydrogenase }\end{array}$ \\
\hline GhUSP & Gh_A03G0386 & $\begin{array}{l}\text { T12 / } \\
\text { T0 }\end{array}$ & 1.20 & 0.37 & $\begin{array}{l}\text { Encodes universal } \\
\text { stress protein }\end{array}$ \\
\hline GhAVP1 & Gh_D05G0122 & $\begin{array}{l}\text { T12 / } \\
\text { T3 }\end{array}$ & 1.42 & 0.62 & $\begin{array}{l}\text { Inorganic } \mathrm{H} \\
\text { pyrophosphatase } \\
\text { family protein }\end{array}$ \\
\hline GhADH1 & Gh_A01G1605 & $\begin{array}{l}\text { T12 / } \\
\text { T3 }\end{array}$ & 2.03 & 0.34 & $\begin{array}{l}\text { Alcohol } \\
\text { dehydrogenase }\end{array}$ \\
\hline
\end{tabular}

\section{Discussion}

There are complex regulatory mechanisms when plants encountered salt stress. Although cotton is the inherently salt-tolerant crop, with the aggravation of soil salinization in its planting area, it is necessary to analysis the molecular mechanism of cotton response to salt stress and cultivate novel cotton varieties with higher salt tolerance. Based on the high yield, perfect fiber quality and disease resistance, Xinluzhong 54 is one of the main cotton variety in Xinjiang nowadays (Additional file 1: Fig. S1a). However, soil salinity is one of the important factors for restricting the further popularization of Xinluzhong 54 (Additional file 1: Fig. S1b and c). Firstly, it is necessary to clarify the molecular mechanism of Xinluzhong 54 in response to salt stress. Here, an integrated analysis strategy of 
transcriptome and proteome was performed to analyze the regulation mechanism of cotton response to salt stress and identify the key regulatory genes which may be used to improve the salt tolerance in cotton.

Plants initiated defense and against salt stress by regulating the expression of stress responsive genes. It has been widely reported that phytohormones were involved in regulating the tolerance of plant response to salt stress (Ryu and Cho 2015). Transcriptome analysis revealed that cotton response to salt stress induced a variety of hormone signal pathway gene, reflecting the complexity of hormone signals involved in the regulation of cotton response to salt stress (Additional file 1: Fig. S6). Some evidences suggested that ABA and JA hormone signals can enhance salt tolerance in many plants (Vishwakarma et al., 2017; Goossens et al., 2016). ABA signal pathway were activated by salt stress, it was consistent with the increase of ABA content in cotton leaves (Li et al., 2019). In addition, some reports have pointed out that JA also plays an important role in regulating salt tolerance in plants, such as rice and wheat (Kang et al., 2005; Qiu et al., 2014). Previous study showed that genes involved in JA synthesis and signal transduction were induced by salt stress, such as $L O X, A O S, J A Z$ and MYC. Appropriate concentrations $\left(0.01 \mu \mathrm{mol} \mathrm{I}^{-1}\right)$ of coronatine, which is structurally and functionally similar to JA, could improve the salt tolerance of cotton (Xie et al., 2015). Overexpression of a JA signal repressor gene GhJAZ2 in cotton, significantly reduces the salt tolerance (Sun et al., 2017). However, cytokinin is different from ABA and JA, it was showed that genes in cytokinin signal pathways were down-regulated, which was consistent with previous report (Liu et al., 2012). We speculated that the inhibition of cytokinin signal may be one of the reasons for repressing cotton growth under salt stress.

Some genes involved in signal transduction were differentially activated or repressed under salt stress, such as transcription factors, CBL interaction protein kinases, MAPK signal cascade pathway (Fig. 4c and d, Additional file 1: Fig. S7). Many transcription factor family genes showed rapid response by salt stress. It was remarkable that most of the differentially expressed NAC genes were up-regulated when cotton encountered salt stress, it is consistent with the previous studies that NAC gene were widely involved in salt response (Sun et al., 2018). Overexpression of a salt stress induced NAC gene GHATAF1, enhances the salt tolerance of cotton (He et al., 2016). Transcriptome analysis showed that CBL interaction protein kinase and MAPK signal genes were induced by salt stress (Fig. 4c and d). In Arabidopsis, CBL2/3 either regulated osmotic and salt stress responses by interacting with CIPK21 and targeting the kinase to the vacuole thus regulating the homeostasis of ions and water on the vacuole (Pandey et al., 2015), or regulated potassium and magnesium ion homeostasis by interacted with CIPK3, CIPK9 and CIPK23 (Liu et al., 2013; Tang et al., 2015). Here, two CBL genes (GhCBL2, GhCBL3), which were homologous to Arabidopsis $C B L 2$ and $C B L 3$, were up-regulated by salt stress, and were predicted to interacted with several salt induced CIPK protein, such as CIPK3, CIPK9 and CIPK23 (Fig. S8a). Arabidopsis CBL2/3. In Arabidopsis, the interaction between MKK2 and MPK4 was involved in regulating salt tolerance (Teige et al., 2004). In cotton, the expression patterns of MAPK cascade genes were significantly induced at 4 hours after salt stress treatment (Chen et al., 2020). In this study, the homologous genes of Arabidopsis 
MKK2 and MPK4 genes were up-regulated by salt stress (Fig. 4d). We speculated that some conservative regulatory mechanisms may exist between cotton and Arabidopsis.

Salt stress induced the accumulation of reactive oxygen species and caused lipid peroxidation. In this study, some antioxidant enzyme genes were up-regulated under salt stress (Fig. 5b), which consistent with the activation of some antioxidant enzymes in previous studies (Meloni et al., 2003). Salt stress induced the increase of $\mathrm{Na}^{+}$content in cotton leaves (Peng et al., 2014). Here, we identified some transporters and channel proteins encoding genes showed differently expressed under salt stress, they might play an important role in regulating $\mathrm{Na}^{+}$homeostasis under salt stress (Fig. $5 \mathrm{c}$ ). Salt stress induced stomatal closure, photosynthetic rate declined, energy metabolism inhibited in plants. Consistent with previous studies, many DEGs and DEPs involved in these biological processes and showed downregulated expression patterns under salt stress.

Our results showed that only 77,70 and 47 differentially expressed genes were associated with mRNA and protein levels in T3 / T0, T12 / T0 and T12 / T3, respectively (Fig. 2a). Previous studies have shown that there was a low correlation between transcriptome and proteome results (Chen et al., 2017; Wang et al., 2015). For example, transcriptional factors, CBL-CIPK and MAPK cascade related genes which were identified in transcriptome were not identified in proteome. The nonlinear and low correlation between these two omics may be due to the following reasons: (i) Post-transcriptional and post-translational modification; (ii) The different rates between transcription and translation, and the different half-life between mRNA and proteins; (iii) The threshold used in the identification of DEGs and DEPs.

However, an integrated analysis of transcriptome and proteome could give more information both on mRNA and protein levels. In the present study, the DEGs which could be simultaneously identified at the transcriptional and protein levels were selected for identifying candidate genes. Based on this research strategy, there were 9 candidate genes which might play an important role in cotton response to salt stress were identified. AtLEA14 (AT1G01470) is the homologous gene of GhLEA14 (Gh_A11G0835) in Arabidopsis, and the amino acid sequence homology between GhLEA14 and AtLEA14 is $70.86 \%$.

Overexpression of AtLEA14 could significantly enhance the salt stress tolerance of Arabidopsis (Jia et al., 2014). We speculate that GhLEA14 is very likely to regulate salt tolerance in cotton. These candidate genes can provide important genetic resources for genetic improvement of salt tolerance in cotton.

\section{Conclusions}

High salt stress could inhibit plant growth, affect production capacity, and even cause plant death. To achieve tolerance and survive under salt stress environment, several changes in transcription and protein levels are aim to maintain osmotic, ionic and ROS homeostasis in cotton. In this study, Xinluzhong 54, a widely cultivated cotton species in Xinjiang, was used as the experimental material to analyze the molecular mechanism of cotton response to salt stress. According to the integrated analysis of transcriptome and proteome, a complex salt stress response network was outlined in cotton (Fig. 6). This 
study can contribute to understand the mechanism of cotton response to salt stress, and facilitate the breeding of new salt-resistant cotton varieties by molecular breeding.

\section{Declarations}

\section{Ethics declarations}

Ethics approval and consent to participate

Not applicable.

Consent for publication

Not applicable.

Availability of data and materials

The datasets used in this study are available from the corresponding author on reasonable request.

\section{Author Contributions}

$\mathrm{CL}$ and $\mathrm{SH}$ performed the experiments, analyzed the data and wrote the manuscript. JK provided the seeds and performed the salt stress assay in the field. $\mathrm{XY}$ and $\mathrm{HX}$ conceived this project and revised the manuscript.

\section{Acknowledgments}

The authors declare that the research was conducted in the absence of any commercial or financial relationships that could be construed as a potential conflict of interest.

\section{Funding}

This work was supported by National R\&D Project of Transgenic Crops of Ministry of Science and Technology of China (2016ZX08005-004-002).

\section{Author information}

Lin Chen and Heng Sun contributed equally to this work.

\section{References}

Al MM, Khan AL, Muneer S. (2020). Silicon in Horticultural Crops: Cross-talk, Signaling, and Tolerance Mechanism under Salinity Stress. Plants. 9 (4). doi:10.3390/plants9040460. 
Chen J, Liu SS, Kohler A, et al. (2017). iTRAQ and RNA-Seq Analyses Provide New Insights into Regulation Mechanism of Symbiotic Germination of Dendrobium officinale Seeds (Orchidaceae). J Proteome Res. 16 (6), 2174-2187. doi:10.1021/acs.jproteome.6b00999.

Chen L, Sun H, Wang FJ, et al. (2020). Genome-wide identification of MAPK cascade genes reveals the GhMAP3K14-GhMKK11-GhMPK31 pathway is involved in the drought response in cotton. Plant Mol Biol. 103(1-2), 211-223. doi:10.1007/s11103-020-00986-0.

Chen QZ, Guo WS, Feng LZ, et al. (2015). Transcriptome and proteome analysis of Eucalyptus infected with Calonectria pseudoreteaudii. J Proteomics. 115, 117-131. doi:10.1016/j.jprot.2014.12.008.

Gong WF, Xu FF, Sun JL, et al. (2017). iTRAQ-Based Comparative Proteomic Analysis of Seedling Leaves of Two Upland Cotton Genotypes Differing in Salt Tolerance. Front Plant Sci. 8, 2113. doi:10.3389/fpls.2017.02113.

Goossens J, Fernandez-Calvo P, Schweizer F, et al. (2016). Jasmonates: signal transduction components and their roles in environmental stress responses. Plant Mol Biol. 91 (6), 673-689. doi:10.1007/s11103016-0480-9

Guo JY, Shi GY, Guo XY, et al. (2015). Transcriptome analysis reveals that distinct metabolic pathways operate in salt-tolerant and salt-sensitive upland cotton varieties subjected to salinity stress. Plant Sci. 238:33-45. doi:10.1016/j.plantsci.2015.05.013.

He X, Zhu LF, Xu L, et al. (2016). GhATAF1., a NAC transcription factor, confers abiotic and biotic stress responses by regulating phytohormonal signaling networks. Plant Cell Rep. 35 (10), 2167-2179. doi:10.1007/s00299-016-2027-6.

Jia FJ, Qi SD, Li H, et al. (2014). Overexpression of Late Embryogenesis Abundant 14 enhances Arabidopsis salt stress tolerance. Biochem Biophys Res Commun. 454 (4), 505-511. doi:10.1016/j.bbrc.2014.10.136.

Kang DJ, Seo YJ, Lee JD, et al. (2005). Jasmonic acid differentially affects growth, ion uptake and abscisic acid concentration in salt-tolerant and salt-sensitive rice cultivars. J Agron Crop Sci. 191 (4), 273282. doi:10.1111/j.1439-037X.2005.00153.x.

Li W, Zhao FA, Fang WP, et al. (2015). Identification of early salt stress responsive proteins in seedling roots of upland cotton (Gossypium hirsutum L.) employing iTRAQ-based proteomic technique. Front Plant Sci. 6:732. doi:10.3389/fpls.2015.00732.

Li Z, Li L, Zhou KH, et al. (2019). GhWRKY6 Acts as a Negative Regulator in Both Transgenic Arabidopsis and Cotton During Drought and Salt Stress. Front Genet. 10:392. doi:10.3389/fgene.2019.00392.

Liu LL, Ren HM, Chen LQ, et al. (2013). A Protein Kinase., Calcineurin B-Like Protein-Interacting Protein Kinase9, Interacts with Calcium Sensor Calcineurin B-Like Protein3 and Regulates Potassium 
Homeostasis under Low-Potassium Stress in Arabidopsis. Plant Physiol. 161 (1), 266-277. doi:10.1104/pp.112.206896.

Liu YD, Yin ZJ, Yu JW, et al. (2012). Improved salt tolerance and delayed leaf senescence in transgenic cotton expressing the Agrobacterium IPT gene. Biologia Plantarum. 56 (2), 237-246. doi:

$10.1007 / \mathrm{s} 10535-012-0082-6$.

Mahajan S, Tuteja N. (2005). Cold, salinity and drought stresses: an overview. Arch Biochem Biophys. 444 (2), 139-158. doi:10.1016/j.abb.2005.10.018.

Meloni DA, Oliva MA, Martinez CA, at al. (2003). Photosynthesis and activity of superoxide dismutase, peroxidase and glutathione reductase in cotton under salt stress. Environ Exp Bot. 49 (1), 69-76. doi:10.1016/s0098-8472(02)00058-8.

Muchate NS, Nikalje GC, Rajurkar NS, et al. (2016). Plant Salt Stress: Adaptive Responses, Tolerance Mechanism and Bioengineering for Salt Tolerance. Bot Rev. 82 (4), 371-406. doi:10.1007/s12229-0169173-y.

Munns R, Tester M. (2008). Mechanisms of salinity tolerance. Annu Rev Plant Biol. 59, 651-681. doi:10.1146/annurev.arplant.59.032607.092911.

Pandey GK, Kanwar P, Singh A, et al. (2015). Calcineurin B-Like Protein-Interacting Protein Kinase CIPK21 Regulates Osmotic and Salt Stress Responses in Arabidopsis. Plant Physiol. 169 (1), 780-792. doi:10.1104/pp.15.00623.

Park HJ, Kim WY, Yun DJ. (2016). A New Insight of Salt Stress Signaling in Plant. Mol Cells. 39 (6), 447459. doi:10.14348/molcells.2016.0083.

Peng Z, He SP, Gong WF, et al. (2014). Comprehensive analysis of differentially expressed genes and transcriptional regulation induced by salt stress in two contrasting cotton genotypes. BMC Genomics. 15 (1), 760. doi:10.1186/1471-2164-15-760.

Peng Z, He SP, Gong WF, et al. (2018). Integration of proteomic and transcriptomic profiles reveals multiple levels of genetic regulation of salt tolerance in cotton. BMC Plant Biol. 18 (1), 128. doi:10.1186/s12870-018-1350-1.

Qiu ZB, Guo JL, Zhu AJ, et al. (2014). Exogenous jasmonic acid can enhance tolerance of wheat seedlings to salt stress. Ecotoxicol Environ Saf. 104, 202-208. doi:10.1016/j.ecoenv.2014.03.014.

Ryu H, Cho YG. (2015). Plant hormones in salt stress tolerance. J Plant Biol. 58 (3), 147-155. doi:10.1007/s12374-015-0103-z.

Sun H, Chen L, Li JY, et al. (2017). The JASMONATE ZIM-domain Gene Family Mediates JA Signaling and Stress Response in Cotton. Plant Cell physiol. 58(12), 2139-2154. doi: 10.1093/pcp/pcx148. 
Sun H, Hu ML, Li JY, et al. (2018). Comprehensive analysis of NAC transcription factors uncovers their roles during fiber development and stress response in cotton. BMC Plant Biol. 18 (1), 150. doi:10.1186/s12870-018-1367-5.

Tang RJ, Zhao FG, Garcia VJ, et al. (2015). Tonoplast CBL-CIPK calcium signaling network regulates magnesium homeostasis in Arabidopsis. Proc Natl Acad Sci U S A. 112 (10), 3134-3139. doi:10.1073/pnas.1420944112.

Teige M, Scheikl E, Eulgem T, et al. (2004). The MKK2 pathway mediates cold and salt stress signaling in Arabidopsis. Mol Cell. 15 (1), 141-152. doi:10.1016/j.molcel.2004.06.023.

Trevisan S, Manoli A, Ravazzolo L, et al. (2015). Nitrate sensing by the maize root apex transition zone: a merged transcriptomic and proteomic survey. J Exp Bot. 66 (13), 3699-3715. doi:10.1093/jxb/erv165.

Vishwakarma K, Upadhyay N, Kumar N, et al. (2017) Abscisic Acid Signaling and Abiotic Stress Tolerance in Plants: A Review on Current Knowledge and Future Prospects. Front Plant Sci. 8:161. doi:10.3389/fpls.2017.00161.

Wang SH, You ZY, Ye LP, et al. (2014). Quantitative Proteomic and Transcriptomic Analyses of Molecular Mechanisms Associated with Low Silk Production in Silkworm Bombyx mori. J Proteome Res. 13 (2), 735-751. doi:10.1021/pr4008333.

Wang XC, Li Q, Jin X, et al. (2015). Quantitative proteomics and transcriptomics reveal key metabolic processes associated with cotton fiber initiation. J Proteomics. 114, 16-27.

doi:10.1016/j.jprot.2014.10.022.

Wang Z, Hong YC, Zhu GT, et al. (2020). Loss of salt tolerance during tomato domestication conferred by variation in a $\mathrm{Na}(+) / \mathrm{K}(+)$ transporter. EMBO J. 39 (10), e103256. doi:10.15252/embj.2019103256.

Wen B, Zhou R, Feng Q, et al. (2014). IQuant: an automated pipeline for quantitative proteomics based upon isobaric tags. Proteomics. 14 (20), 2280-2285. doi:10.1002/pmic.201300361.

Xie C, Mao XZ, Huang JJ, et al. (2011). KOBAS 2.0: a web server for annotation and identification of enriched pathways and diseases. Nucleic Acids Res. 39 (Web Server issue), W316-322. doi:10.1093/nar/gkr483.

Xie ZX, Duan LS, Li ZH, et al. (2015). Dose-Dependent Effects of Coronatine on Cotton Seedling Growth Under Salt Stress. J Plant Growth Regul. 34 (3), 651-664. doi:10.1007/s00344-015-9501-1.

Yang YQ, Guo Y. (2018). Elucidating the molecular mechanisms mediating plant salt-stress responses. New phytol. 217 (2), 523-539. doi:10.1111/nph.14920.

Zelm EV, Zhang YX, Testerink C. (2020). Salt Tolerance Mechanisms of Plants. Annu Rev Plant Biol. 71, 403-433. doi:10.1146/annurev-arplant-050718100005. 
Zhang M, Liang XY, Wang LM, et al. (2019). A HAK family $\mathrm{Na}(+)$ transporter confers natural variation of salt tolerance in maize. Nat Plants. 5 (12), 1297-1308. doi:10.1038/s41477-019-0565-y.

Zhang TZ, Hu Y, Jiang WK, et al. (2015). Sequencing of allotetraploid cotton (Gossypium hirsutum L. acc. TM-1) provides a resource for fiber improvement. Nat Biotechnol. 33 (5), 531-537. doi:10.1038/nbt.3207.

Zorb C, Geilfus CM, Dietz KJ. (2019). Salinity and crop yield. Plant Biol (Stuttg). 1, 31-38. doi:10.1111/plb.12884.

\section{Figures}

A

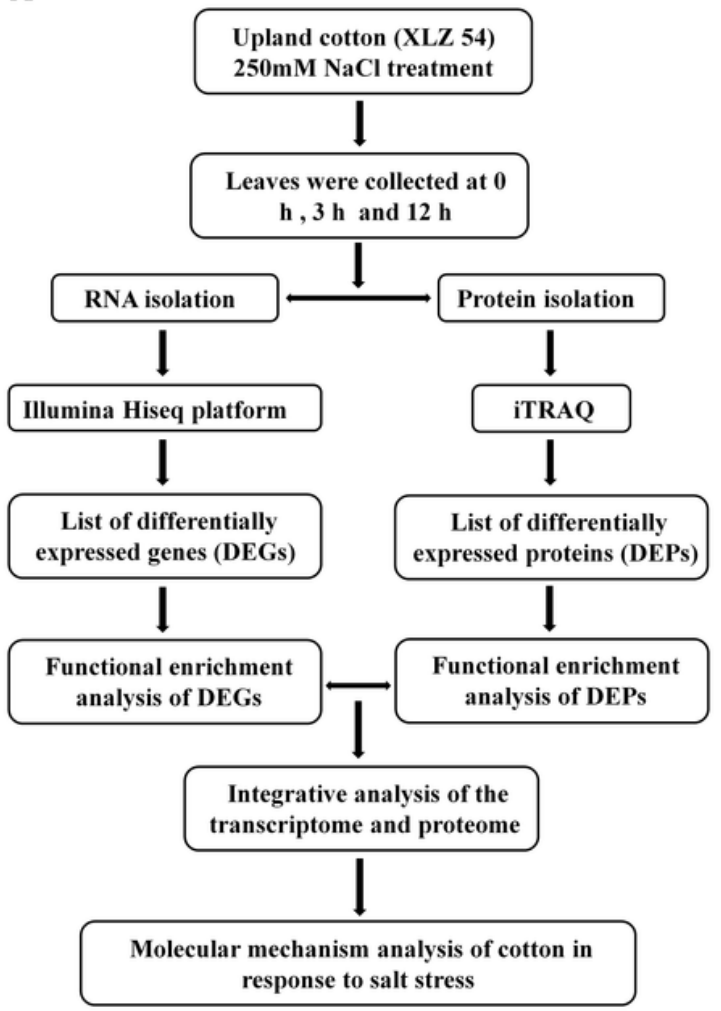

B

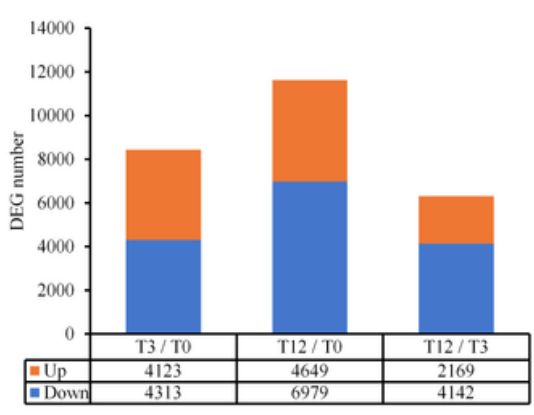

C

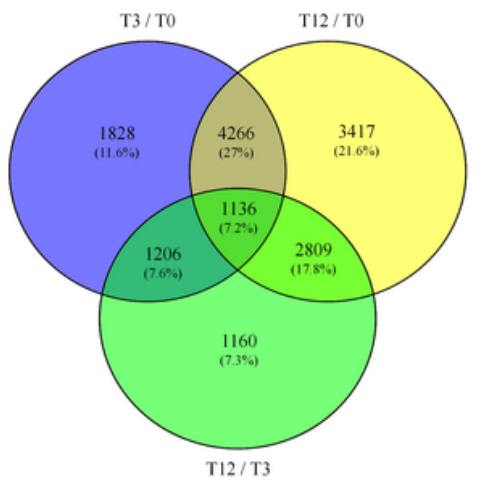

D

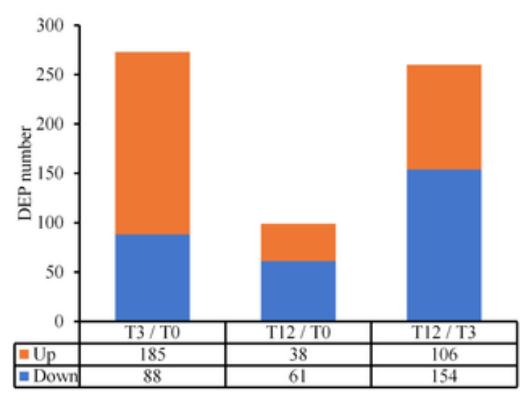

E

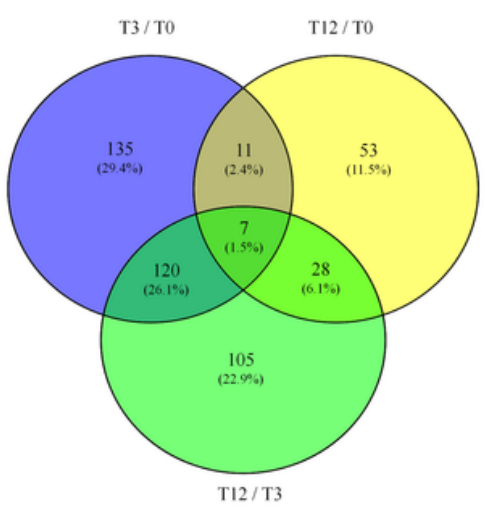

Figure 1

Identification of differentially expressed genes (DEGs) in response to salt stress. a Schematic of experimental in integrated transcriptome and proteome analysis. b Overview of genome-wide gene expression under salt stress. $c$ The number of DEGs in different comparison groups. $d$ The number of DEGs on each chromosome. e qRT-PCR validation of transcript levels evaluated by RNA-Seq. 
A

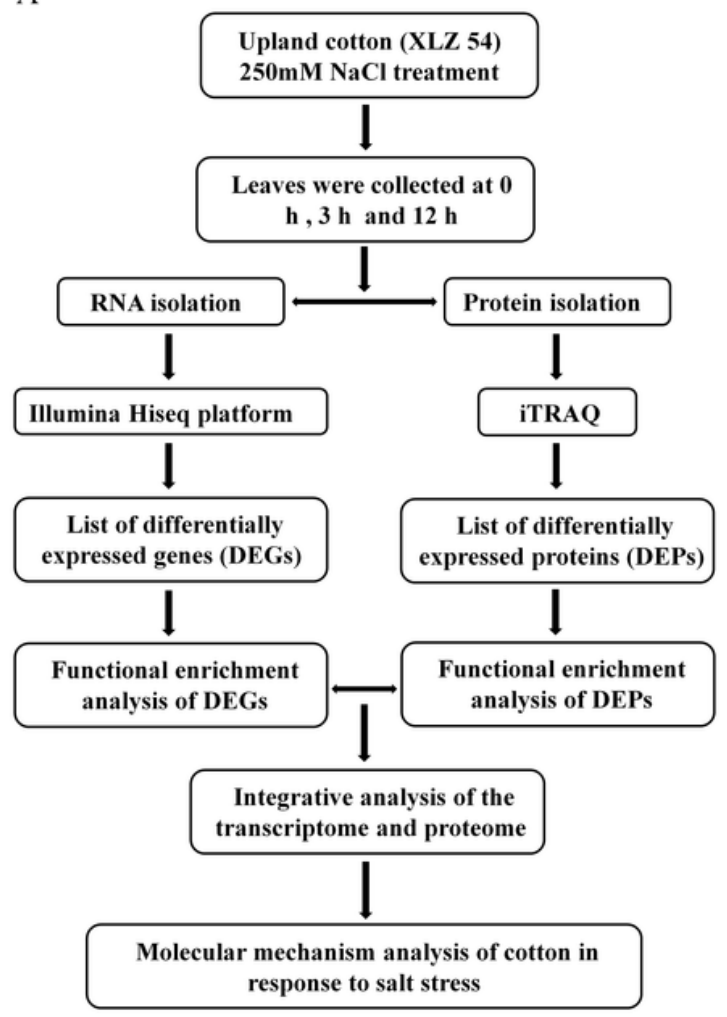

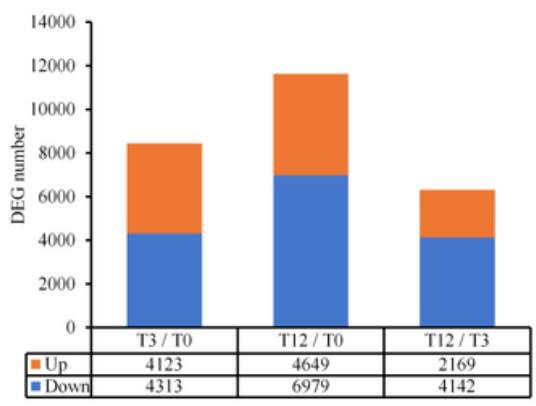

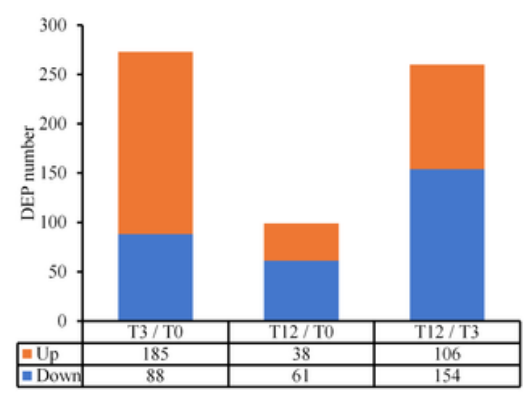

C

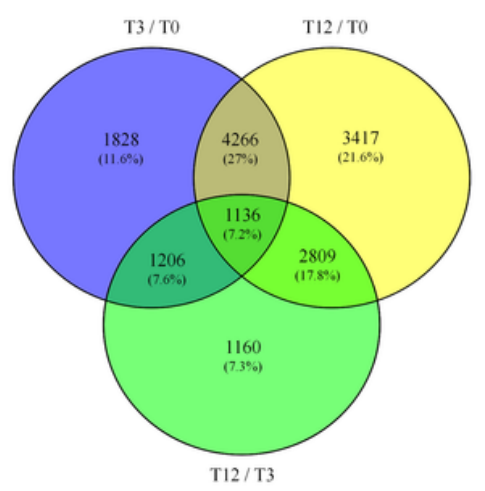

E

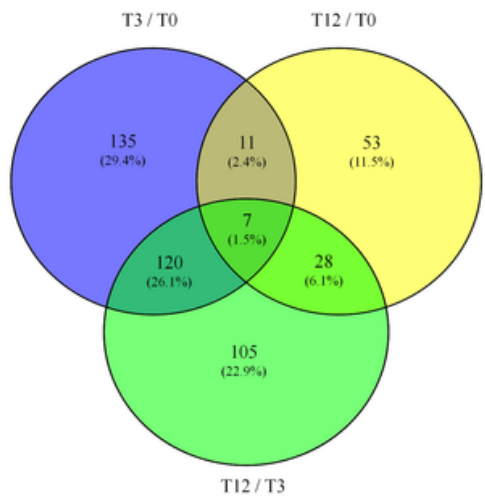

\section{Figure 1}

Identification of differentially expressed genes (DEGs) in response to salt stress. a Schematic of experimental in integrated transcriptome and proteome analysis. b Overview of genome-wide gene expression under salt stress. $c$ The number of DEGs in different comparison groups. $d$ The number of DEGs on each chromosome. e qRT-PCR validation of transcript levels evaluated by RNA-Seq.
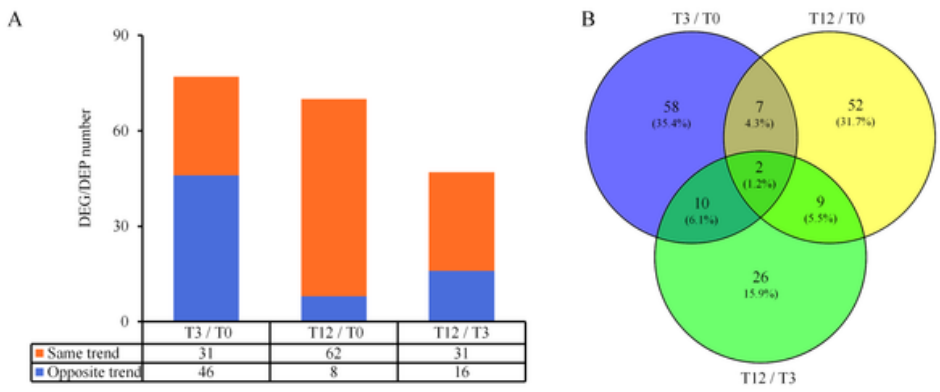

$\mathrm{C}$
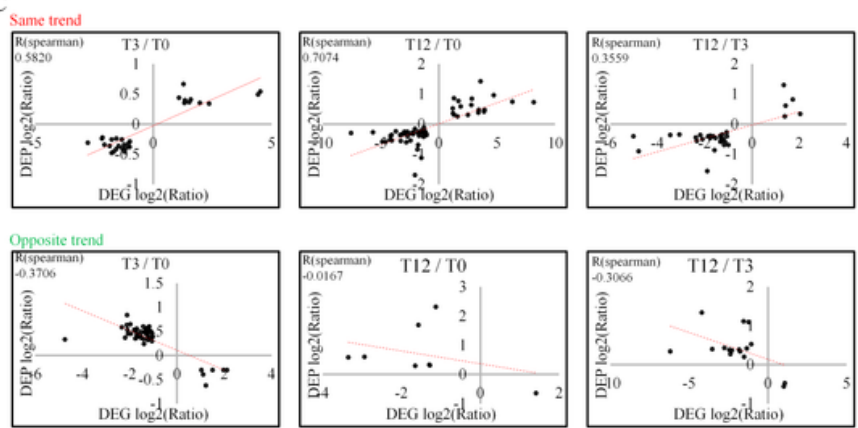

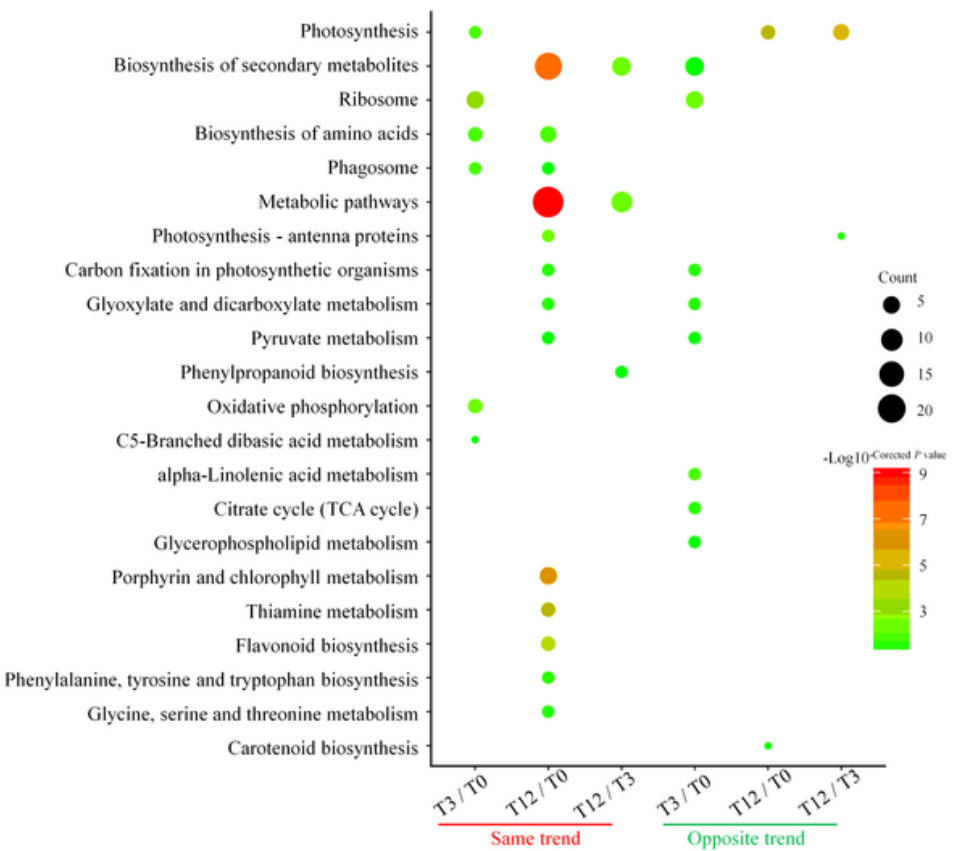


Figure 2

Statistics and functional analysis on the correlation between DEGs and DEPs. a Statistics on the number of correlated DEGs and DEPs. b Venn diagram of correlated DEGs/DEPs. c Concordance between the gene changes at transcriptome and proteome levels. $d$ Functional enrichment analysis of the correlated DEGs and DEPs.

A

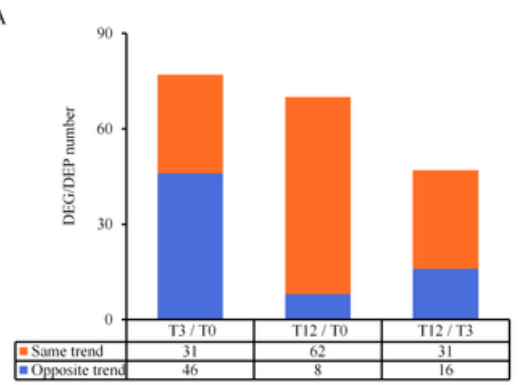

C
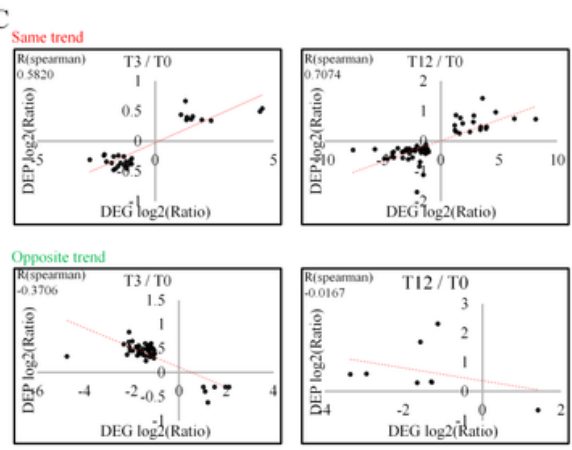
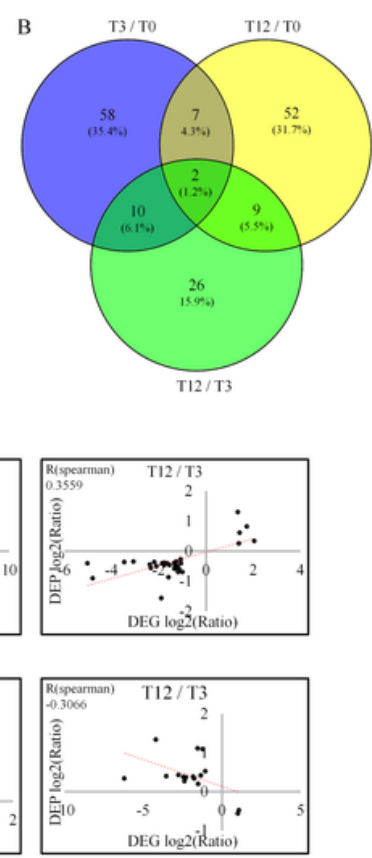
Carbon fixation in photosynthetic organisms Glyoxylate and dicarboxylate metabolism Pyruvate metabolism Phenylpropanoid biosynthesis Oxidative phosphorylation C5-Branched dibasic acid metabolism alpha-Linolenic acid metabolism Citrate cycle (TCA cycle) Glycerophospholipid metabolism Porphyrin and chlorophyll metabolism Thiamine metabolism Flavonoid biosynthesis Phenylalanine, tyrosine and tryptophan biosynthesis Glycine, serine and threonine metabolism Carotenoid biosynthesis

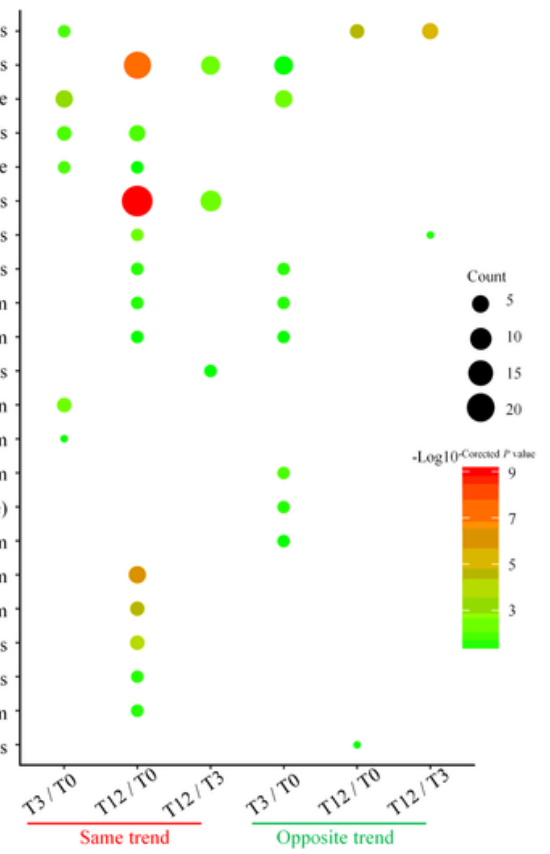

Figure 2

Statistics and functional analysis on the correlation between DEGs and DEPs. a Statistics on the number of correlated DEGs and DEPs. b Venn diagram of correlated DEGs/DEPs. c Concordance between the gene changes at transcriptome and proteome levels. $d$ Functional enrichment analysis of the correlated DEGs and DEPs. 
A

GhPFK3

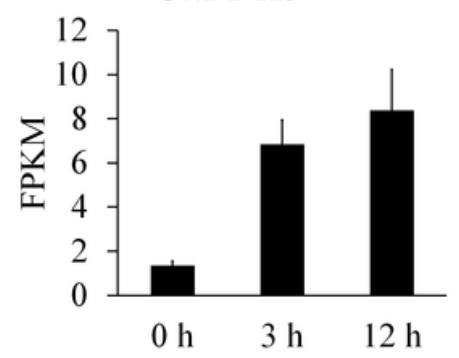

B

C

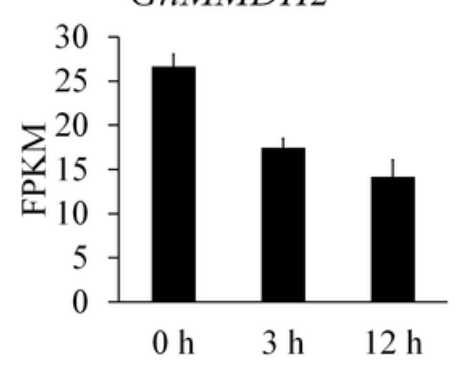

GhLHCB2

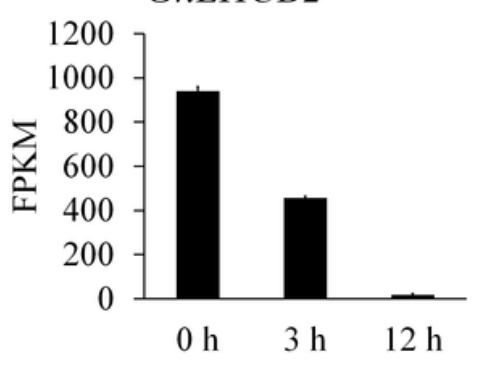

GhPKP2

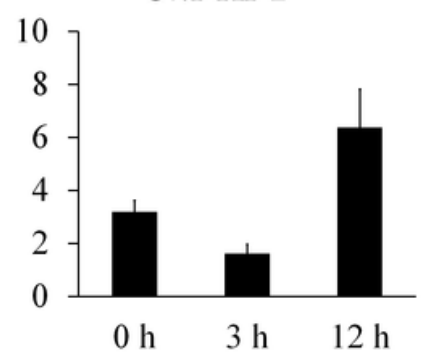

GhMMDH1

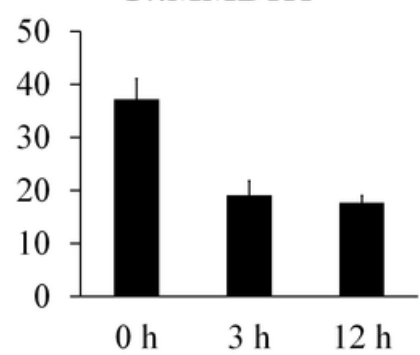

GhLHCA4

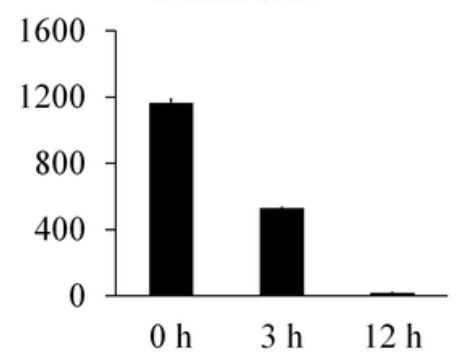

GhPKPl

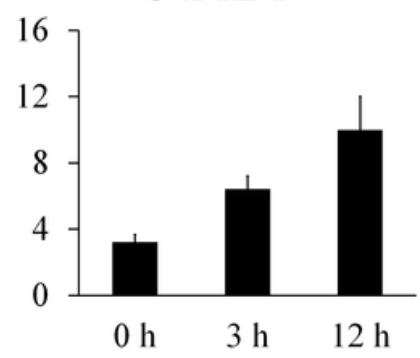

GhCICDH

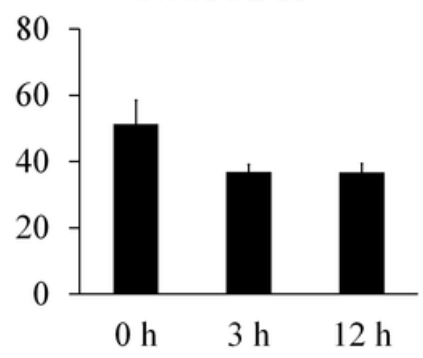

GhLHCBI

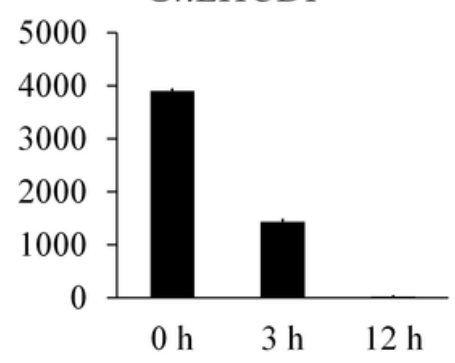

GhHXK3

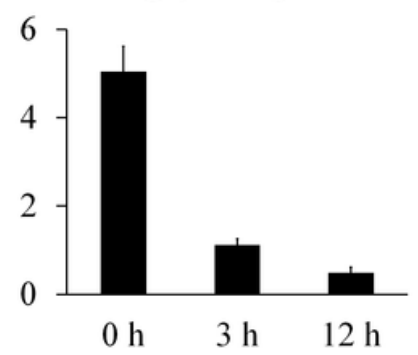

GhMAB1

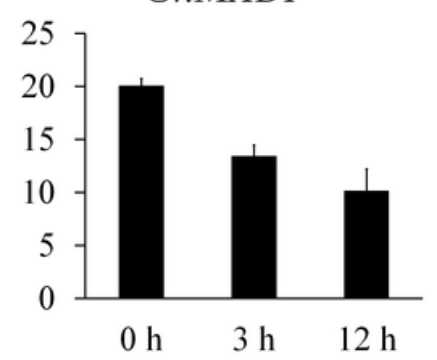

GhPSAO

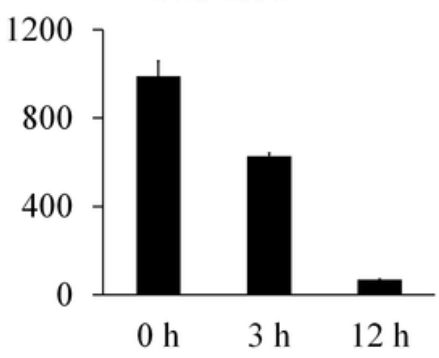

\section{Figure 3}

The expression patterns of function genes which were involved in energy metabolism. a The expression patterns of genes involved in the glycolysis/ gluconeogenesis pathways. b The expression patterns of genes involved in tricarboxylic acid cycle. c The expression patterns of genes involved in photosynthesis. 
A

GhPFK3

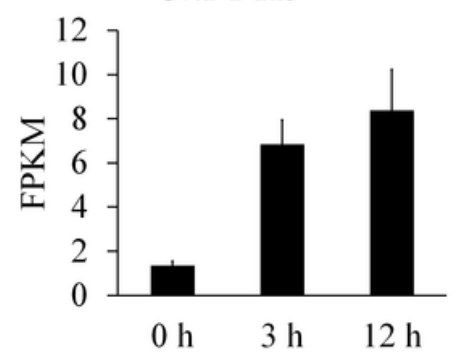

B

C

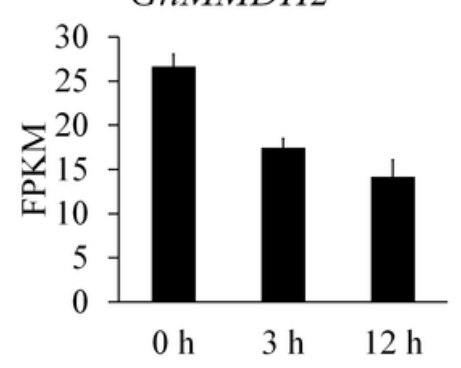

GhLHCB2

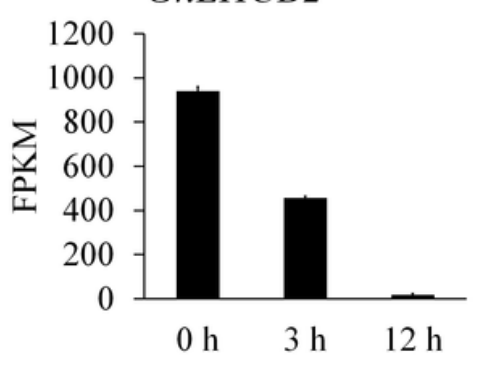

GhPKP2

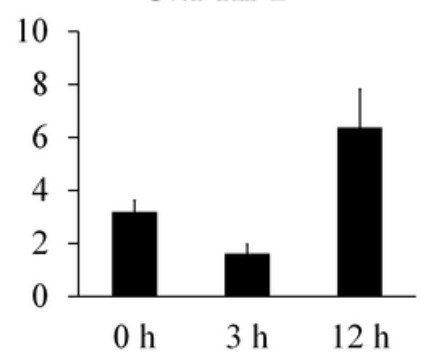

GhMMDH1

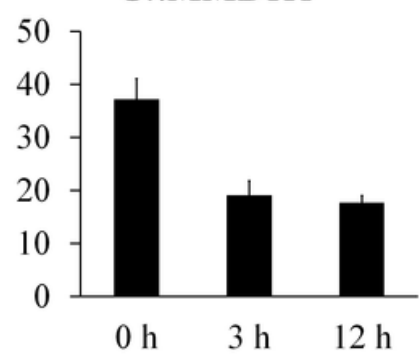

GhLHCA4

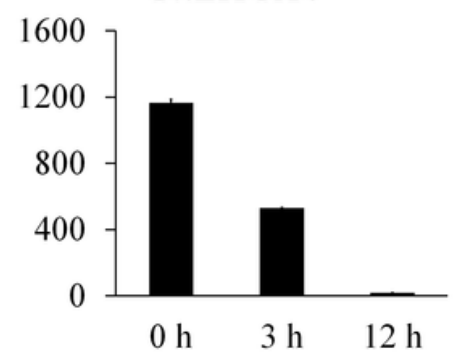

GhPKPI

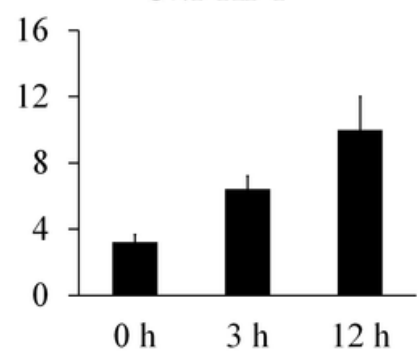

GhCICDH

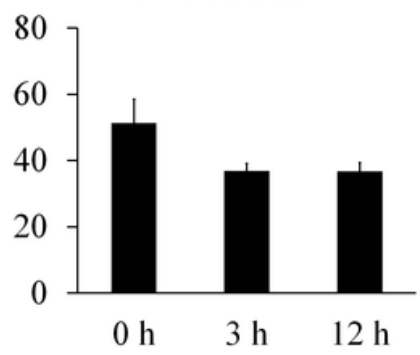

GhLHCBI

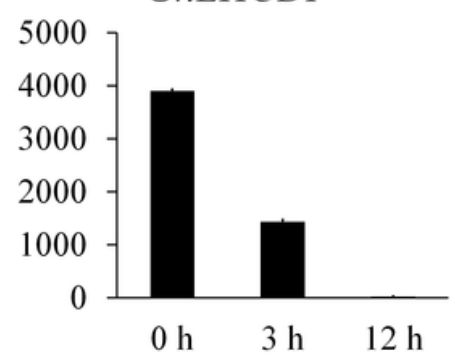

GhHXK3

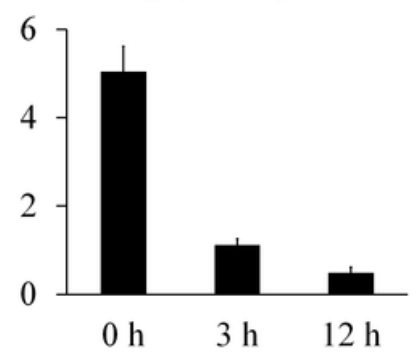

GhMAB1

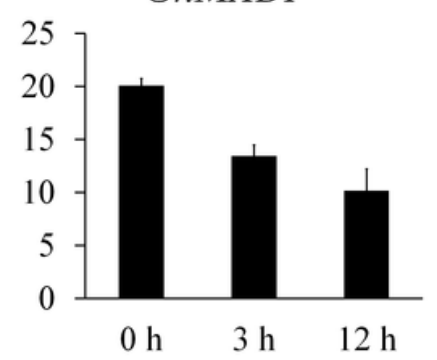

GhPSAO

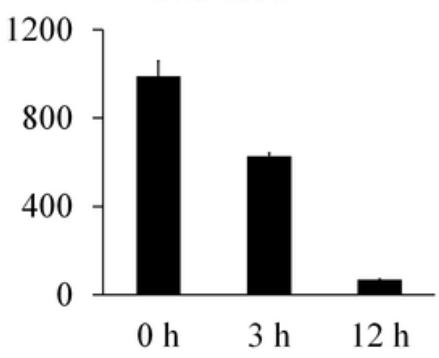

\section{Figure 3}

The expression patterns of function genes which were involved in energy metabolism. a The expression patterns of genes involved in the glycolysis/ gluconeogenesis pathways. b The expression patterns of genes involved in tricarboxylic acid cycle. c The expression patterns of genes involved in photosynthesis. 

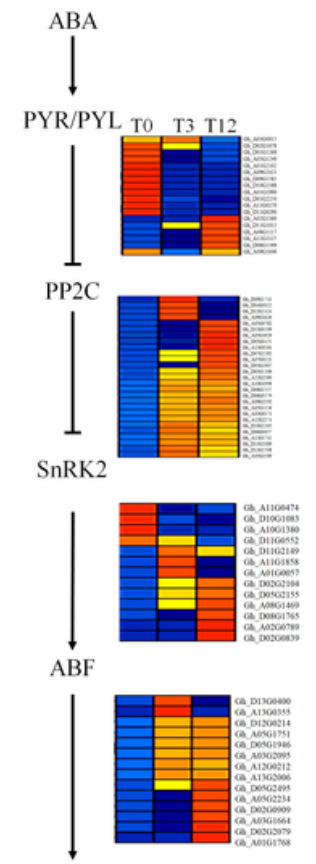

Stress response

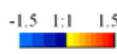

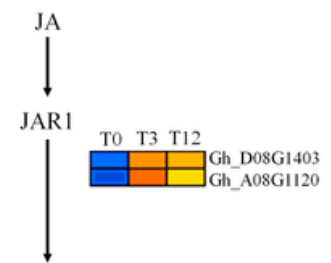
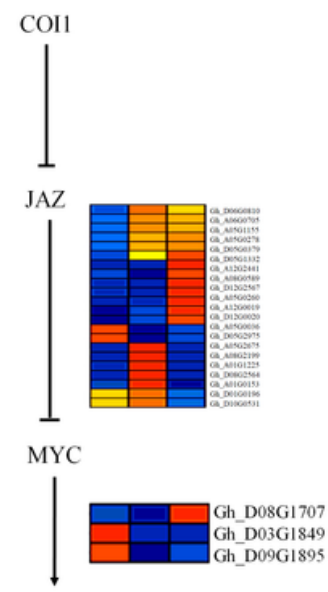

Stress response

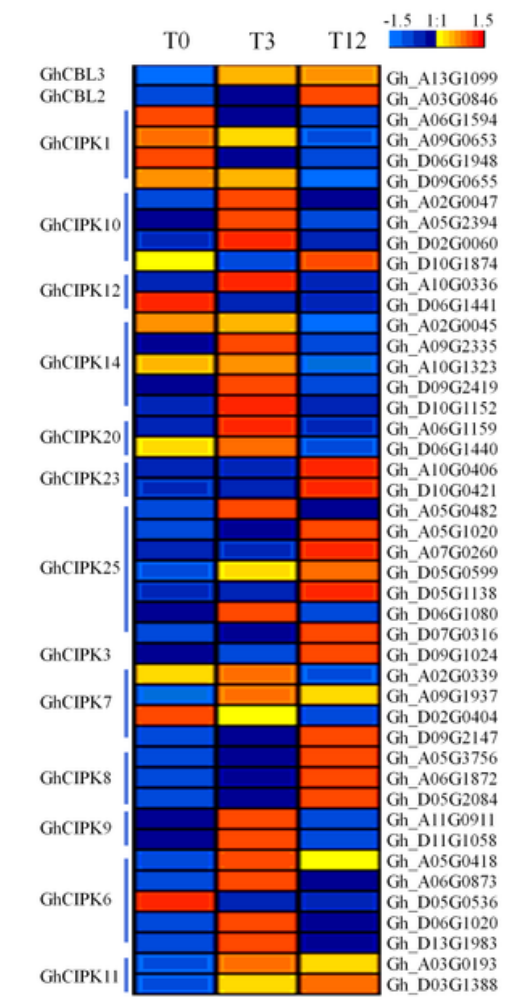

D

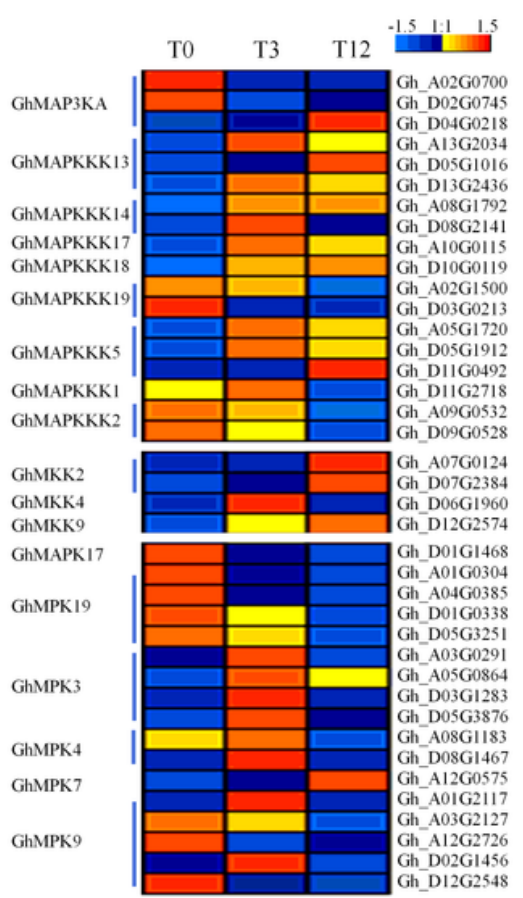

Figure 4

Plant signal transduction in response to salt stress in cotton. $a-b$ The expression patterns of genes which were involved in ABA and JAZ signal pathway. c Expression patterns of CBL and CIPK genes. $d$ Expression patterns of MAPK signal cascade genes. 

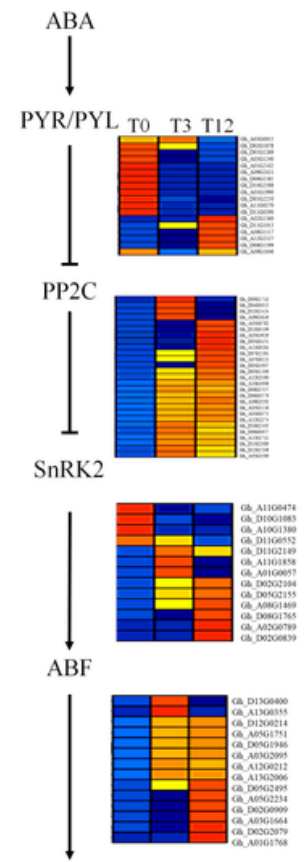

Stress response

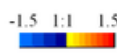

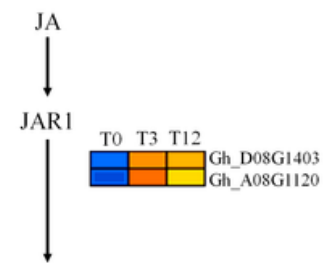
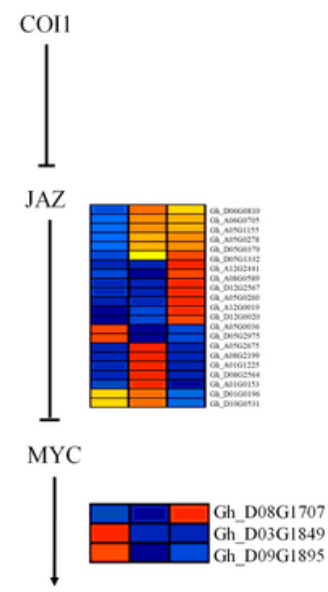

Stress response

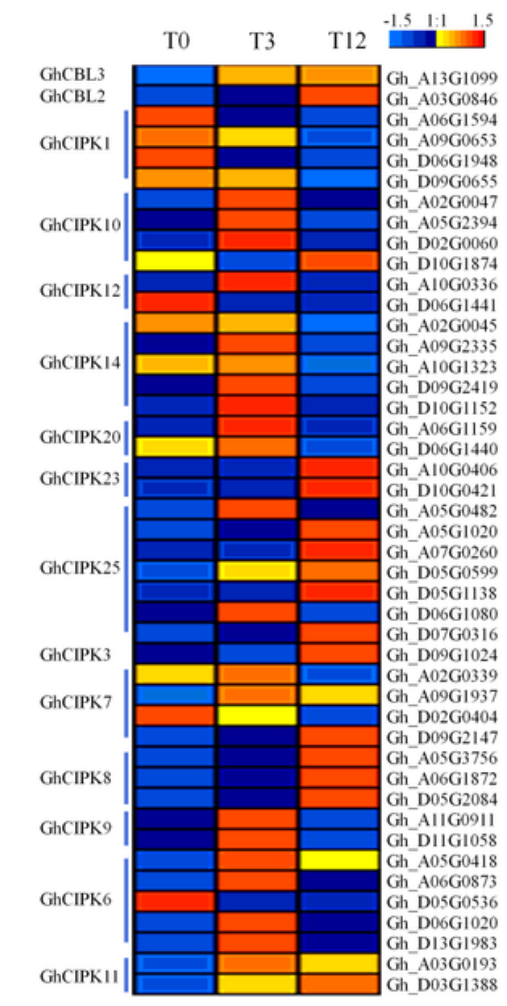

D

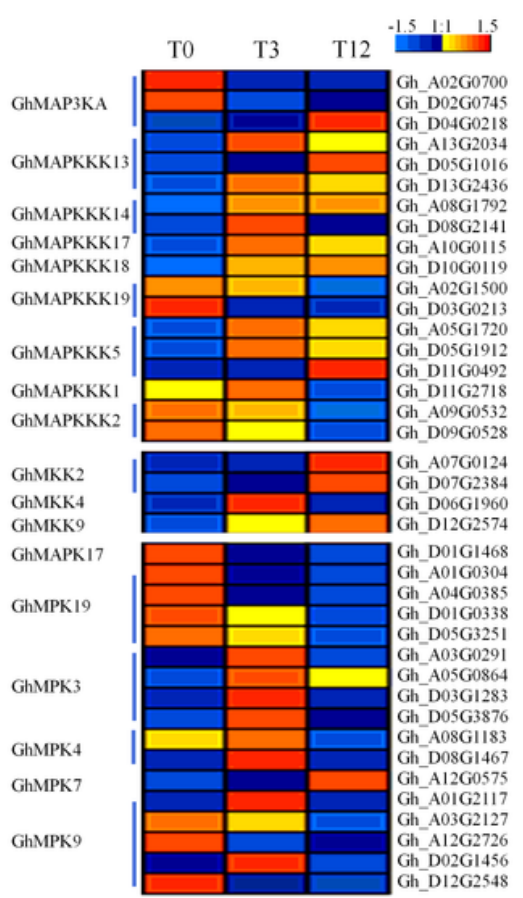

Figure 4

Plant signal transduction in response to salt stress in cotton. $a-b$ The expression patterns of genes which were involved in ABA and JAZ signal pathway. c Expression patterns of CBL and CIPK genes. $d$ Expression patterns of MAPK signal cascade genes. 
A
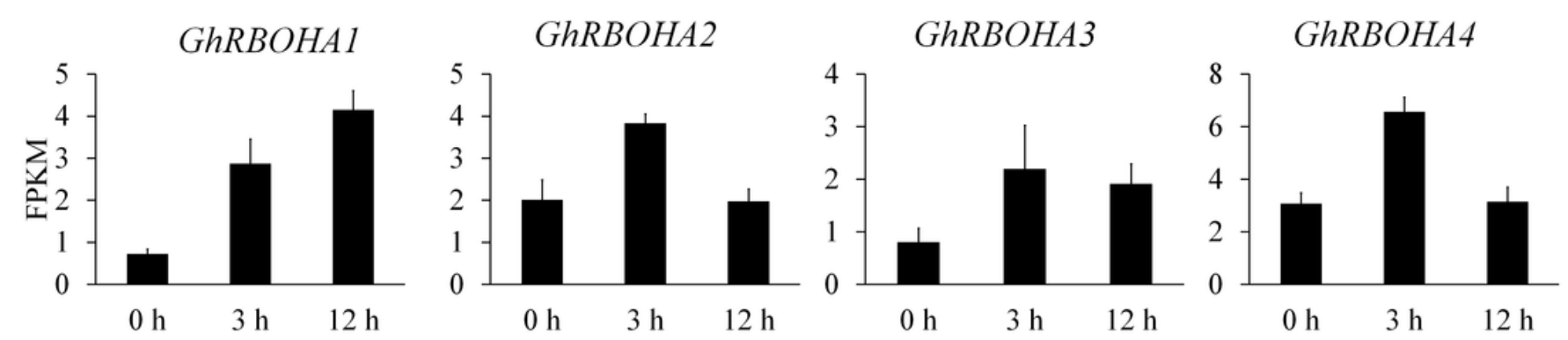

B
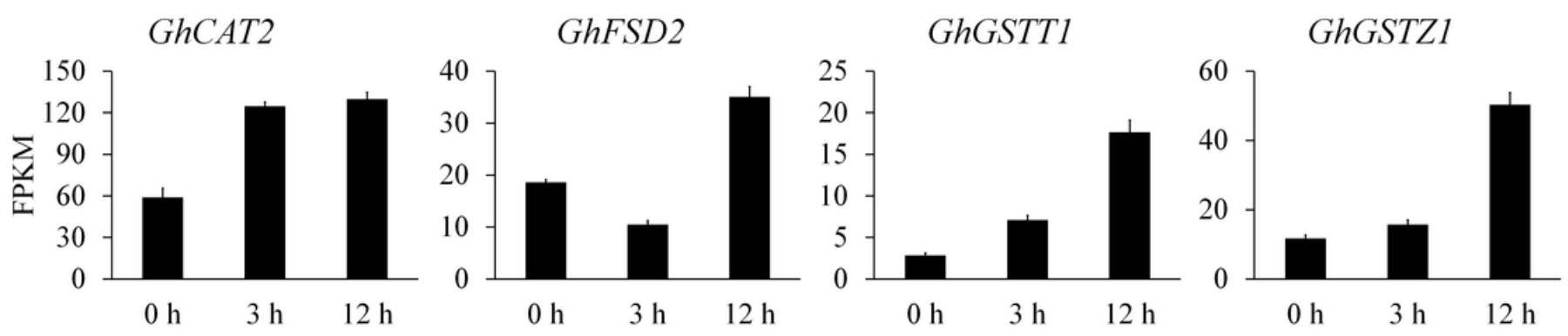

$\mathrm{C}$
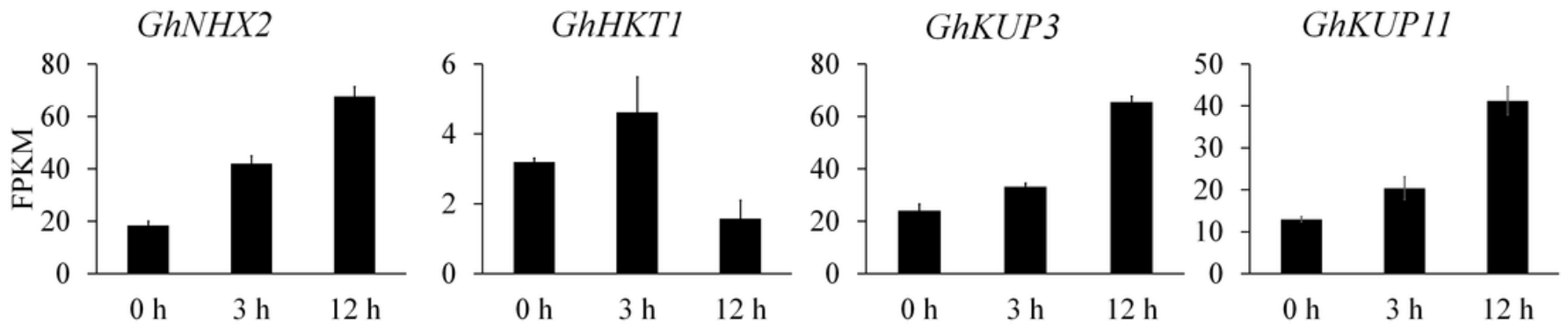

$\mathrm{D}$

GhTPK1

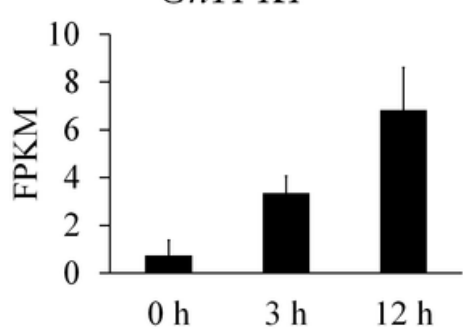

GhAKT1

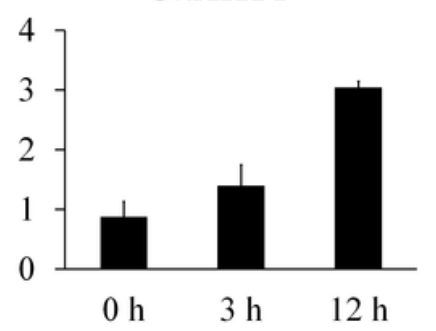

GhSKOR

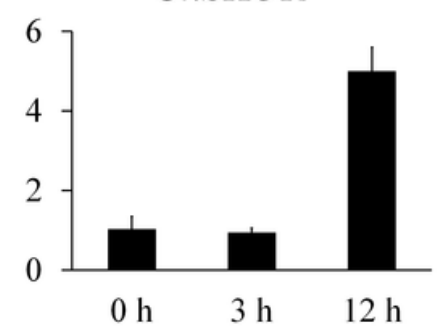

GhKCO5

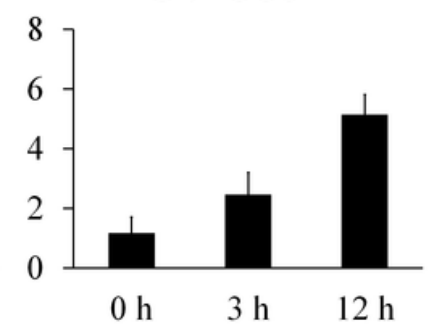

Figure 5

The expression patterns of genes involved in Antioxidant and ion transport systems. a-b Expression patterns of genes related to reactive oxygen species production and scavenging. c-d Expression patterns of ion transport related genes. 
A
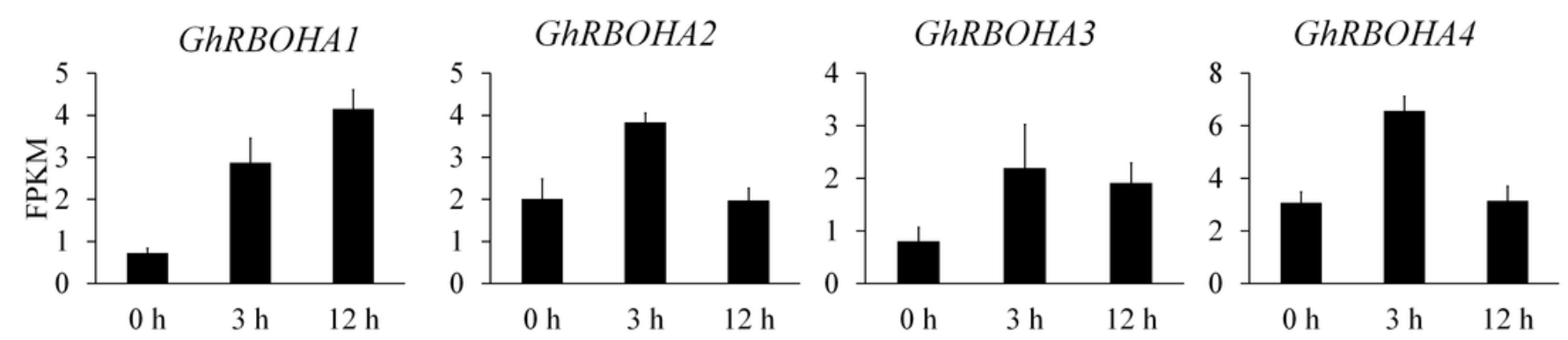

B
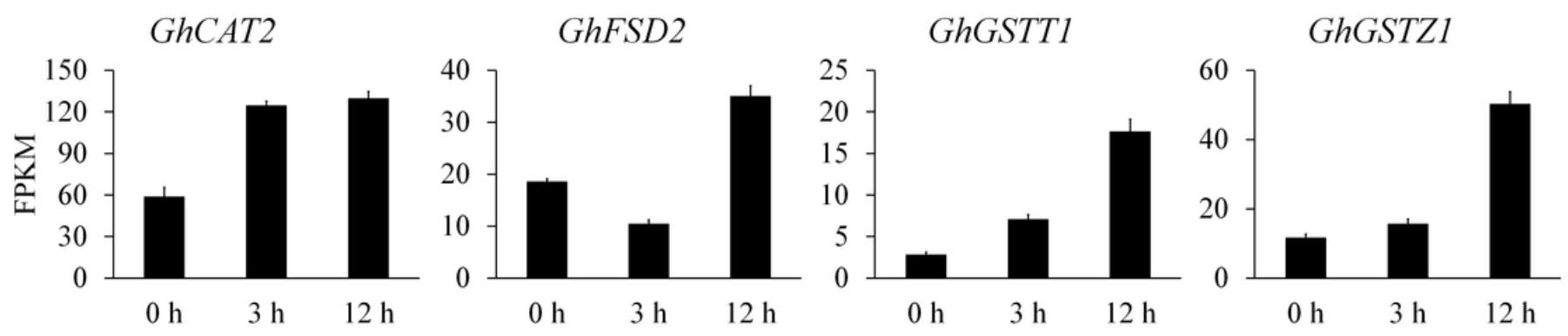

$\mathrm{C}$
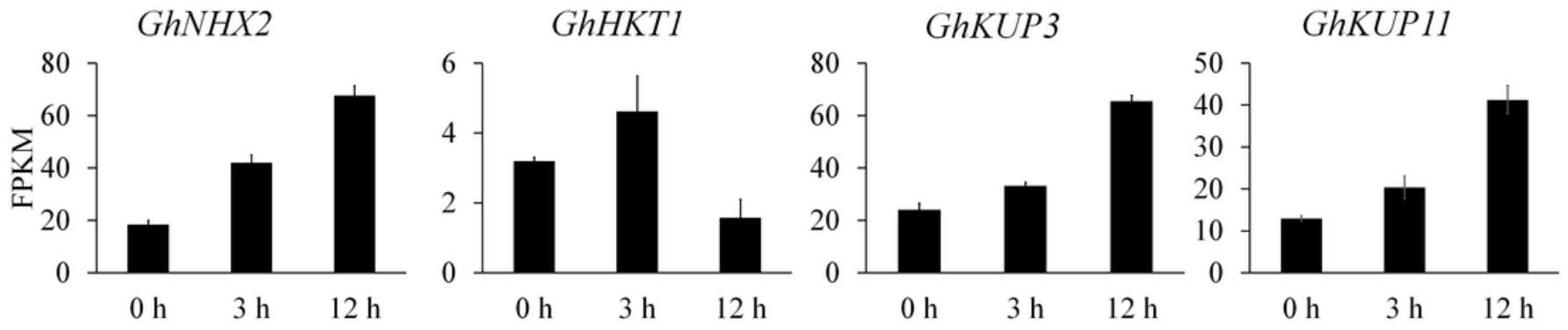

$\mathrm{D}$

GhTPK1

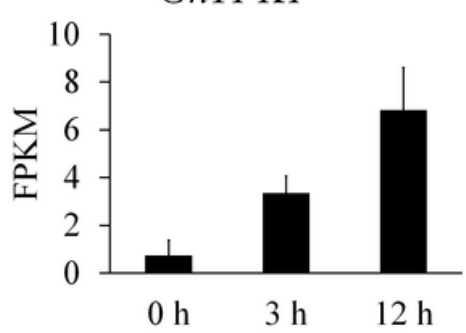

GhAKT1

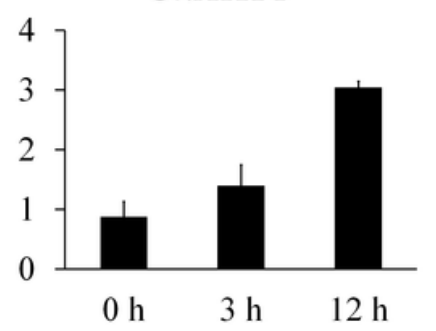

GhSKOR

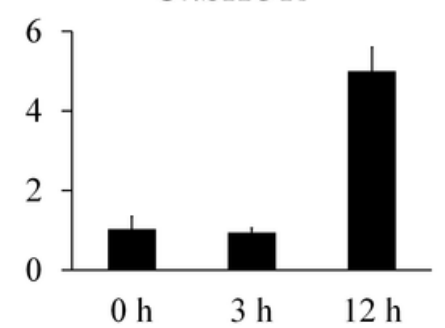

GhKCO5

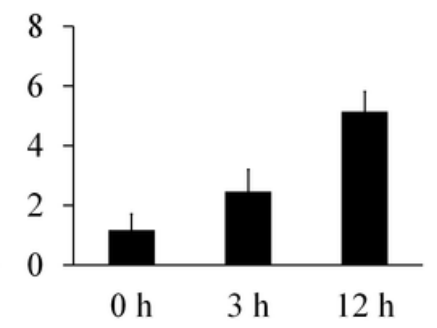

Figure 5

The expression patterns of genes involved in Antioxidant and ion transport systems. a-b Expression patterns of genes related to reactive oxygen species production and scavenging. c-d Expression patterns of ion transport related genes. 


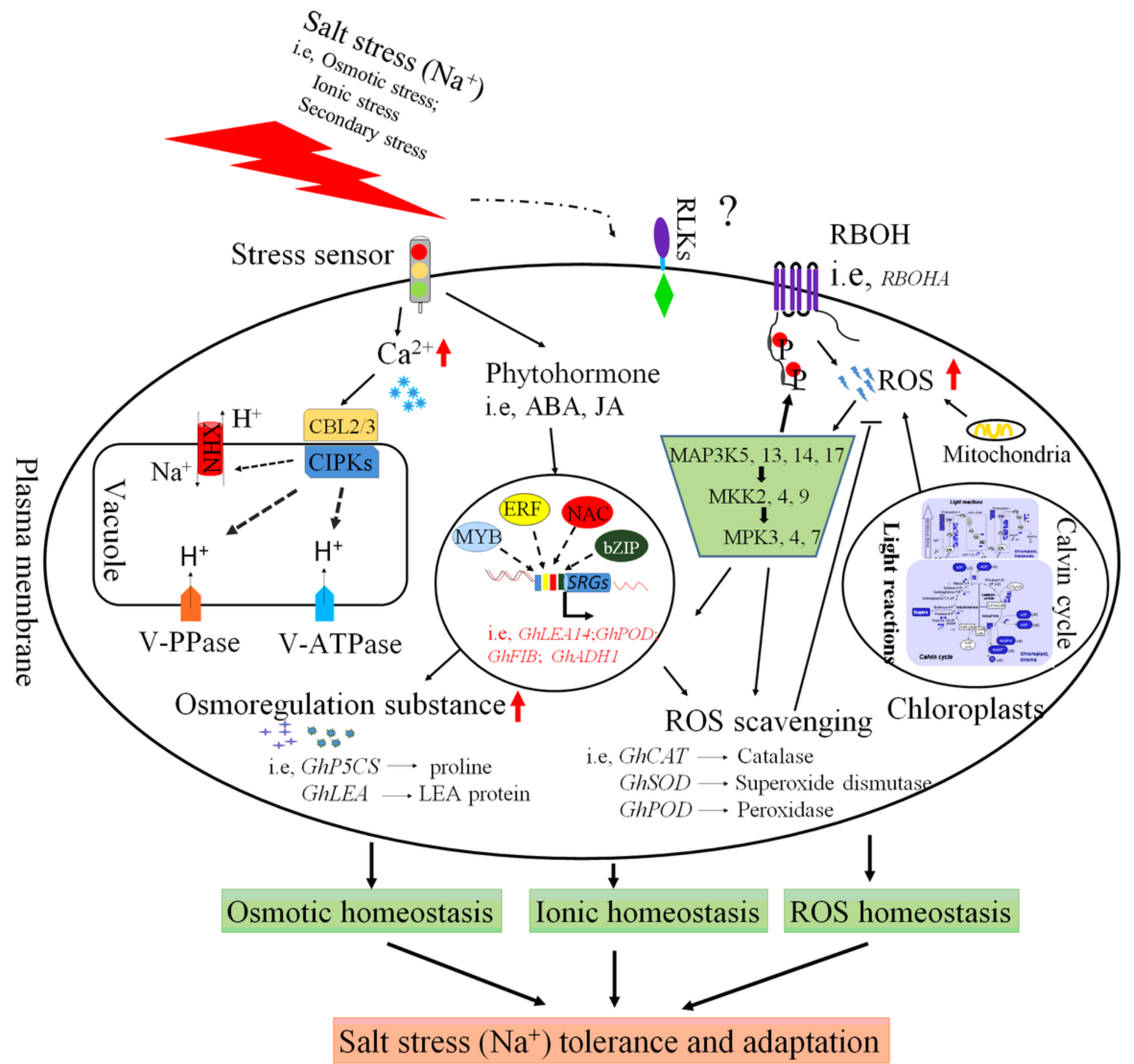

Figure 6

Schematic presentation of a possible regulatory model in cotton (Gossypium hirsutum L. acc. Xinluzhong 54) leaves under salt stress. Cells of cotton sense salt stress by RLKs and other unknown sensors, then transfer external signal into intracellular, and change the content of $\mathrm{Ca} 2+, \mathrm{ROS}$ and other hormones (i.e, ABA, JA). Inside the cell, Ca2+ signal cascade (i.e, GhCBL2,3; GhCIPK8,9,10,25), ROS, MAPK cascade (i.e, GhMAP3K5,13,14,17-GhMKK2,4,9-GhMPK3,4,7) and other hormone signal cascades pathway are activated, which could alter the global transcriptional profiles in cotton (the expression of stress related transcription factors, such as MYB, ERF, NAC and bZIP are initiated). The expression of genes encoding proteins that related to salt stress response, including $\mathrm{NHX}$, osmoregulation related and ROS scavenging 
proteins (i.e, GhLEA14; GhP5CS; GhPOD; GhSOD; GhCAT; GhFIB; GhADH1). Ultimately, the osmotic homeostasis, ionic homeostasis and ROS homeostasis pathways are activated to facilitate cotton to resist and adapt to salt stress.

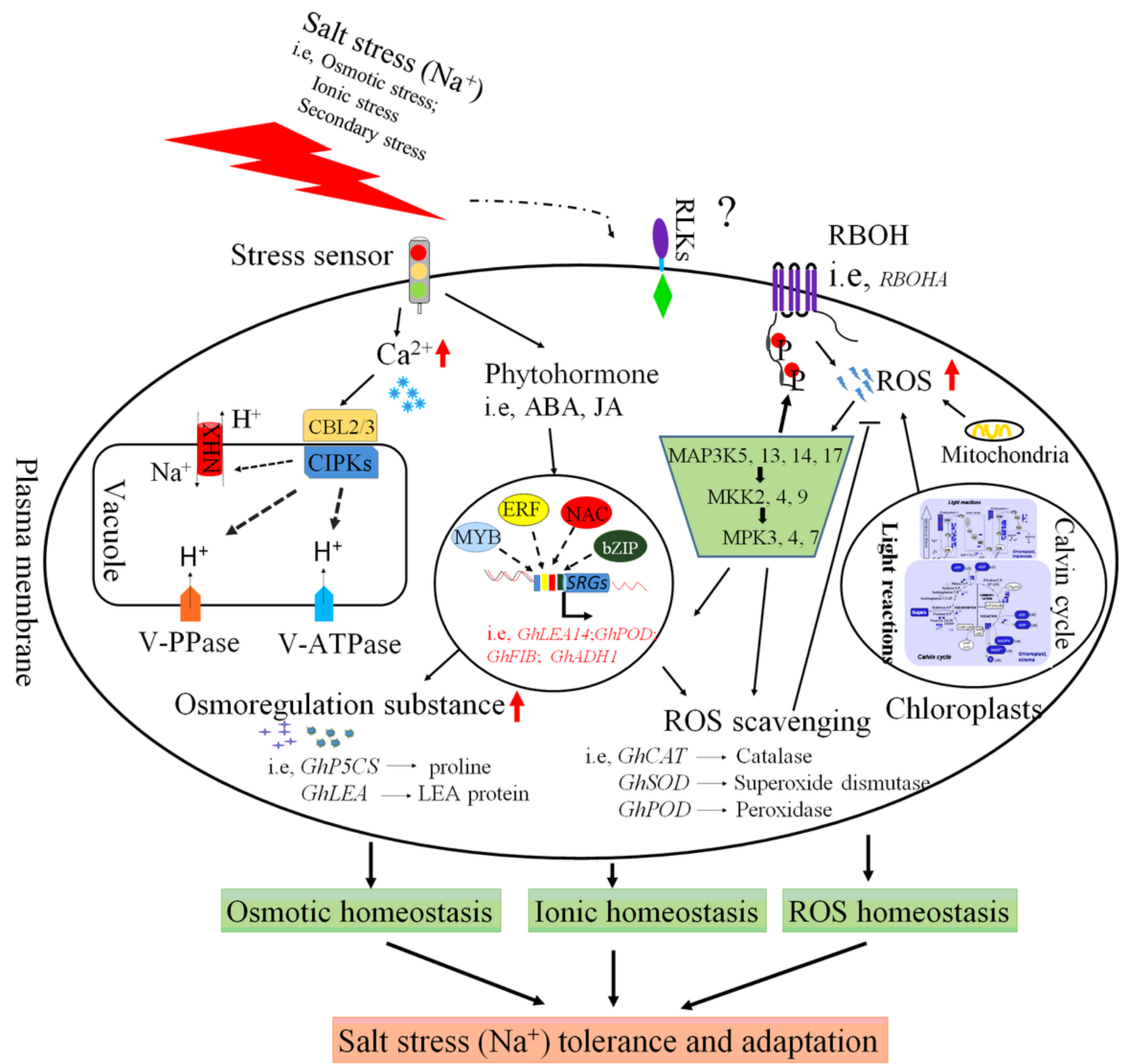

Figure 6

Schematic presentation of a possible regulatory model in cotton (Gossypium hirsutum L. acc. Xinluzhong 54) leaves under salt stress. Cells of cotton sense salt stress by RLKs and other unknown sensors, then transfer external signal into intracellular, and change the content of $\mathrm{Ca} 2+$, ROS and other hormones (i.e, ABA, JA). Inside the cell, Ca2+ signal cascade (i.e, GhCBL2,3; GhCIPK8,9,10,25), ROS, MAPK cascade (i.e, GhMAP3K5,13,14,17-GhMKK2,4,9-GhMPK3,4,7) and other hormone signal cascades pathway are 
activated, which could alter the global transcriptional profiles in cotton (the expression of stress related transcription factors, such as MYB, ERF, NAC and bZIP are initiated). The expression of genes encoding proteins that related to salt stress response, including $\mathrm{NHX}$, osmoregulation related and ROS scavenging proteins (i.e, GhLEA14; GhP5CS; GhPOD; GhSOD; GhCAT; GhFIB; GhADH1). Ultimately, the osmotic homeostasis, ionic homeostasis and ROS homeostasis pathways are activated to facilitate cotton to resist and adapt to salt stress.

\section{Supplementary Files}

This is a list of supplementary files associated with this preprint. Click to download.

- Additionalfile1.rar

- Additionalfile1.rar 